MATHEMATICS OF COMPUTATION

Volume 68, Number 228, Pages 1347-1378

S 0025-5718(99)01125-4

Article electronically published on May 19, 1999

\title{
A COMPARISON OF A POSTERIORI ERROR ESTIMATORS FOR MIXED FINITE ELEMENT DISCRETIZATIONS BY RAVIART-THOMAS ELEMENTS
}

\author{
BARBARA I. WOHLMUTH AND RONALD H.W. HOPPE
}

\begin{abstract}
We consider mixed finite element discretizations of linear second order elliptic boundary value problems with respect to an adaptively generated hierarchy of possibly highly nonuniform simplicial triangulations. In particular, we present and analyze four different kinds of error estimators: a residual based estimator, a hierarchical one, error estimators relying on the solution of local subproblems and on a superconvergence result, respectively. Finally, we examine the relationship between the presented error estimators and compare their local components.
\end{abstract}

\section{INTRODUCTION}

We consider the following boundary value problem for a linear second order elliptic differential operator

$$
\begin{aligned}
u:=-\operatorname{div}(a \nabla u)+b u & =f \quad \text { in } \Omega, \\
u & =0 \quad \text { on } \Gamma:=\partial \Omega,
\end{aligned}
$$

where $\Omega$ stands for a bounded, polygonal domain in the Euclidean space $\mathbb{R}^{2}$ and $f \in L^{2}(\Omega)$. Furthermore, we assume $a=\left(a_{i j}\right)_{i, j=1}^{2}$ to be a symmetric, matrixvalued function with $a_{i j} \in L^{\infty}(\Omega), 1 \leq i, j \leq 2$, and $b \in L^{\infty}(\Omega)$ satisfying

$$
\begin{gathered}
\alpha_{0}|\xi|^{2} \leq \sum_{i, j=1}^{2} a_{i j}(x) \xi_{i} \xi_{j} \leq \alpha_{1}|\xi|^{2}, \\
\xi \in \mathbb{R}^{2}, 0<\alpha_{0} \leq \alpha_{1}, 0 \leq b_{0} \leq b(x) \leq b_{1},
\end{gathered}
$$

for almost all $x \in \Omega$. The local bounds on a subset $D \subset \Omega$ are denoted by $\alpha_{i}^{D}$, $\beta_{i}^{D}, 0 \leq i \leq 1$. For simplicity, we have chosen homogeneous Dirichlet boundary data, but all subsequent results can be easily applied to more general boundary conditions.

In many applications, the flux $\mathbf{j}:=-a \nabla u$ is more important than the primal variable $u$. Therefore, the original problem (1.1) is transformed into a first order system by introducing the auxiliary variable $\mathbf{j}$. The natural ansatz space for the flux is

$$
H(\operatorname{div} ; \Omega):=\left\{\mathbf{q} \in\left(L^{2}(\Omega)\right)^{2} \mid \operatorname{div} \mathbf{q} \in L^{2}(\Omega)\right\},
$$

Received by the editor September 6, 1996 and, in revised form, August 11, 1997 and January 6, 1998.

1991 Mathematics Subject Classification. Primary 65F10, 65N30, 65N50, 65N55.

Key words and phrases. Mixed finite elements, a posteriori error estimation, adaptive grid refinement.

(C)1999 American Mathematical Society 
which is a Hilbert space with respect to the inner product

$$
(\mathbf{p}, \mathbf{q})_{\operatorname{div}}:=(\mathbf{p}, \mathbf{q})_{0}+(\operatorname{div} \mathbf{p}, \operatorname{div} \mathbf{q})_{0}
$$

and the associated norm $\|\cdot\|_{\text {div }}:=(\cdot, \cdot)_{\text {div }}^{1 / 2}$. As usual we denote by $(\cdot, \cdot)_{k}, k \geq 0$, the standard inner product on $H^{k}(\Omega)$ and $\left(H^{k}(\Omega)\right)^{2}$, while $|\cdot|_{k},\|\cdot\|_{k}$ stand for the associated seminorms and norms, respectively.

Then, the weak formulation associated with (1.1) gives rise to the following saddle point problem:

Find $(\mathbf{j}, u) \in H(\operatorname{div} ; \Omega) \times L^{2}(\Omega)$ such that

$$
\begin{aligned}
a(\mathbf{j}, \mathbf{q})+b(\mathbf{q}, u) & =0, & & \mathbf{q} \in H(\operatorname{div} ; \Omega), \\
b(\mathbf{j}, v)-c(u, v) & =-l(v), & & v \in L^{2}(\Omega),
\end{aligned}
$$

where the bilinear forms $a(\cdot, \cdot), b(\cdot, \cdot), c(\cdot, \cdot)$ and the functional $l(\cdot)$ are given by

$$
\begin{aligned}
a(\mathbf{p}, \mathbf{q}) & :=\int_{\Omega} a^{-1} \mathbf{p} \cdot \mathbf{q} d x, \quad \mathbf{p}, \mathbf{q} \in H(\operatorname{div} ; \Omega), \\
b(\mathbf{q}, v) & :=-\int_{\Omega} \operatorname{div} \mathbf{q} v d x, \quad \mathbf{q} \in H(\operatorname{div} ; \Omega), v \in L^{2}(\Omega), \\
c(u, v) & :=\int_{\Omega} b u v d x, \quad u, v \in L^{2}(\Omega), \\
l(v) & :=\int_{\Omega} f v d x, \quad v \in L^{2}(\Omega) .
\end{aligned}
$$

The existence and uniqueness of the solution of system (1.3) are well known (cf. e.g. [12, $\S I I$, Thm. 1.2)]. Associated with the bilinear form $a(\cdot, \cdot)$ is the norm $\|\cdot\|_{\text {div }}$, where $\|\mathbf{q}\|_{\text {div }}^{2}:=\int_{\Omega} a^{-1} \mathbf{q q} d x+\int_{\Omega} \operatorname{div} \mathbf{q} \operatorname{div} \mathbf{q} d x$. Compared with the norm $\|\cdot\|_{\text {div }}$, the norm $\|\cdot\|_{\text {div }}$ is weighted by $a^{-1}$ and plays the same role as the energy norm in the primal formulation. Both norms are equivalent due to the positive definiteness of $a$.

The mixed finite element approach is based on (1.3). Here, we use RaviartThomas finite elements with respect to a simplicial triangulation $\mathcal{T}_{h}$ of $\Omega$. The sets of vertices and edges are denoted by $\mathcal{P}_{h}:=\mathcal{P}_{h}^{0} \cup \mathcal{P}_{h}^{\Gamma}, \mathcal{E}_{h}:=\mathcal{E}_{h}^{0} \cup \mathcal{E}_{h}^{\Gamma}$ where $\mathcal{P}_{h}^{0}, \mathcal{E}_{h}^{0}$ refer to the interior vertices and edges and $\mathcal{P}_{h}^{\Gamma}, \mathcal{E}_{h}^{\Gamma}$ to those located on $\Gamma=\partial \Omega$. Furthermore, $P_{k}(D), D \subseteq \Omega, k \geq 0$, stands for the set of polynomials of degree $\leq k$ on $\mathrm{D}$.

For the discretization of the flux $\mathbf{j} \in H(\operatorname{div} ; \Omega)$ we choose the Raviart-Thomas ansatz space

$$
R T_{k}\left(\Omega ; \mathcal{T}_{h}\right):=\left\{\mathbf{q}_{h} \in H(\operatorname{div} ; \Omega)\left|\mathbf{q}_{h}\right|_{T} \in R T_{k}(T), T \in \mathcal{T}_{h}\right\},
$$

where $R T_{k}(T), T \in \mathcal{T}_{h}$, stands for the Raviart-Thomas element

$$
R T_{k}(T):=P_{k}(T)^{2}+P_{k}(T) \mathbf{x}, \quad \mathbf{x}=\left(x_{1}, x_{2}\right)^{T} .
$$

The degrees of freedom of $R T_{k}(T)$ are given by the following moments:

$$
\int_{e_{i}} \mathbf{n} \cdot \mathbf{q}_{h} p d \sigma, p \in P_{k}\left(e_{i}\right), \quad \int_{T} \mathbf{p} \cdot \mathbf{q}_{h} d x, \mathbf{p} \in P_{k-1}(T),
$$

where $\mathbf{n}$ is the outer normal on $\partial T$ and $e_{i}, 1 \leq i \leq 3$, are the edges of $T$. The discrete ansatz space for the primal variable $u \in L^{2}(\Omega)$ associated with $R T_{k}\left(\Omega ; \mathcal{T}_{h}\right)$ is given by piecewise polynomial functions:

$$
W_{k}\left(\Omega ; \mathcal{T}_{h}\right):=\left\{v_{h} \in L^{2}(\Omega)\left|v_{h}\right|_{T} \in P_{k}(T), T \in \mathcal{T}_{h}\right\} .
$$


Thus, the natural requirement $\operatorname{div} R T_{k}\left(\Omega ; \mathcal{T}_{h}\right)=W_{k}\left(\Omega ; \mathcal{T}_{h}\right)$ is guaranteed. Now, the lowest order mixed discretization of system (1.3) can be written as follows:

Find $\left(\mathbf{j}_{h}, u_{h}\right) \in R T_{0}\left(\Omega ; \mathcal{T}_{h}\right) \times W_{0}\left(\Omega ; \mathcal{T}_{h}\right)$ such that the following discrete saddle point problem is satisfied

$$
\begin{aligned}
a\left(\mathbf{j}_{h}, \mathbf{q}_{h}\right)+b\left(\mathbf{q}_{h}, u_{h}\right) & =0, & & \mathbf{q}_{h} \in R T_{0}\left(\Omega ; \mathcal{T}_{h}\right), \\
b\left(\mathbf{j}_{h}, v_{h}\right)-c\left(u_{h}, v_{h}\right) & =-l\left(v_{h}\right), & & v_{h} \in W_{0}\left(\Omega ; \mathcal{T}_{h}\right) .
\end{aligned}
$$

Note that the Babuška-Brezzi condition is fulfilled and that system (1.4) admits a unique solution (cf., e.g., [12, §II, Prop. 2.11)]).

Throughout the following we refer to $(\mathbf{j}, u) \in H(\operatorname{div} ; \Omega) \times L^{2}(\Omega)$ as the unique solution of the mixed variational problem (1.3) and to $\left(\mathbf{j}_{h}, u_{h}\right) \in R T_{0}\left(\Omega ; \mathcal{T}_{h}\right) \times$ $W_{0}\left(\Omega ; \mathcal{T}_{h}\right)$ as the lowest order Raviart-Thomas approximation satisfying (1.4). Further, we denote by $\left(\tilde{\mathbf{J}}_{h}, \tilde{u}_{h}\right) \in R T_{0}\left(\Omega ; \mathcal{T}_{h}\right) \times W_{0}\left(\Omega ; \mathcal{T}_{h}\right)$ an available computed approximation obtained by means of an appropriate iterative solution process.

In particular, we advocate multilevel iterative solvers that work on a hierarchy $\left(\mathcal{T}_{k}\right)_{k=0}^{j}$ of simplicial triangulations of $\Omega$ generated by the well known refinement process due to Bank et al. [5]. The refinement strategy is such that a triangle $T \in \mathcal{T}_{k}, k \geq 0$, either remains unrefined, or is subdivided into four congruent subtriangles, or is bisected into two subtriangles. Following the refinement rules in [5], each triangle $T \in \mathcal{T}_{k}, k \geq 0$, is geometrically similar either to an element of $\mathcal{T}_{0}$ or to a bisected triangle of $\mathcal{T}_{0}$. The diameter of $T, T \in \mathcal{T}_{k}$, is denoted by $h_{T}$, and $h_{e}$ stands for the length of the edge $e \in \mathcal{E}_{k}$. Then, the regularity of the sequence $\left(\mathcal{T}_{k}\right)_{k=0}^{j}$ guarantees the existence of constants $0<\kappa_{0} \leq \kappa_{1}$ such that

$$
\kappa_{0} h_{e_{i}}^{2} \leq|T| \leq \kappa_{1} h_{e_{i}}^{2}, \quad 1 \leq i \leq 3, \quad T \in \mathcal{T}_{k},
$$

where $|T|$ is the area of $T$. Moreover, due to the local quasiuniformity of $\left(\mathcal{T}_{k}\right)_{k=0}^{j}$ there exist constants $\kappa_{D}, \kappa_{K}>0$ such that for $T \in \mathcal{T}_{k}$

$$
\operatorname{card}\left\{e \in \mathcal{E}_{k}, e \cap \partial T \neq \emptyset\right\} \leq \kappa_{K}, \quad \operatorname{card}\left\{T^{\prime} \in \mathcal{T}_{k}, \partial T^{\prime} \cap \partial T \neq \emptyset\right\} \leq \kappa_{D} .
$$

We assume that the iterative approximation $\left(\tilde{\mathbf{J}}_{h}, \tilde{u}_{h}\right) \in R T_{0}\left(\Omega ; \mathcal{T}_{k}\right) \times W_{0}\left(\Omega ; \mathcal{T}_{k}\right)$ satisfies the second equation of the discrete saddle point problem exactly. This can be achieved, for instance, by using the algorithm proposed by Ewing and Wang [17] for a vanishing Helmholtz term $b \equiv 0$, which was later generalized in [19] and [31] to the case of nonvanishing $b$.

Reliable and efficient a posteriori error estimators are an indispensable tool for efficient adaptive algorithms. We refer to the pioneering work done by Babuška and Rheinboldt [3, 4], Eriksson, Johnson and Hansbo [16, 22, 23], and to the recent survey articles by Bornemann et al. [8] and Verfürth [27, 30] (cf., e.g., [22, 26, 33]). Following the classification of Verfürth [27], we generalize the standard concepts for a posteriori error estimators and present four different types. In the mixed setting, it takes an extra effort to develop an adequate error estimator compared with the standard primal formulation.

We shall derive a posteriori error estimators for the total error $\mathbf{e}_{\mathbf{j}}:=\mathbf{j}-\tilde{\mathbf{j}}_{h}$ in the flux measured in a weighted norm of the flux space $H(\operatorname{div} ; \Omega)$, the total error $e_{u}:=u-\tilde{u}_{h}$ in the primal variable measured in the $L^{2}$-norm, and the total error in both the flux and the primal variable. Denoting the total error to be estimated by $\epsilon$, an estimator $\eta$ is said to be efficient if there exists a constant $\gamma>0$, independent of the refinement level such that $\gamma \eta \leq \epsilon$, whereas $\eta$ is called reliable if there exists another constant $\Gamma \geq \gamma$, independent of the refinement level, such that $\epsilon \leq \Gamma \eta$. In 
this paper, we shall consider a posteriori error estimators that are both efficient and reliable, i.e., estimators satisfying

$$
\gamma \eta \leq \epsilon \leq \Gamma \eta
$$

The paper is organized as follows:

In Section 2, the different types of a posteriori error estimators are introduced and the main results of this paper are summarized. For details we refer to Sections 3-6, where the error estimators are discussed thoroughly and upper and lower bounds for the total error are established.

In Section 3, we investigate a residual based error estimator. This kind of error estimator is based on the dual norm of the residual (cf., e.g., [3, 4, 7, 28, 27, 29]). Due to a Helmholtz decomposition of the ansatz space for the flux into subspaces of solenoidal and weakly irrotational vector fields, the corresponding continuous defect problem can be split into two independent subproblems. The first subproblem is associated with the divergence free part of the flux space and can be treated as in the conforming primal formulation, whereas the second subproblem gives rise to an indefinite saddle point problem.

In Sections 4 and 5, we present two types of hierarchical error estimators that are strongly related and require an adequate saturation assumption (cf., e.g., [6, $14,15,21,25,27])$. In particular, for the derivation of the first hierarchical error estimator, which is dealt with in Section 4, we start from an approximation of the defect problem in a higher order ansatz space followed by a localization in terms of an appropriate hierarchical two-level splitting. For the construction of the other hierarchical error estimator, in Section 5 we proceed the other way around and begin with a suitable localization of the defect problem involving local subproblems that are solved by a hierarchical splitting of an elementwise higher order ansatz. In each case, we propose an estimator for the $H(\operatorname{div} ; \Omega)$-norm of the error in the flux variable, as well as an estimator for the combined error in the flux and in the primal variable.

In Section 6, we consider an error estimator for the primal variable in the $L^{2}$ norm. This error estimator is motivated by a superconvergence result for the finite element approximation of $u$. It is obtained by a comparison of the piecewise constant approximation of the primal variable with a higher order finite element solution arising from a modified nonconforming approach. Finally, we show that the difference between the piecewise constant and the nonconforming approximation is equivalent to a formulation that can be obtained by using some local averaging techniques (cf., e.g., [11, 24, 32, 34]).

In Section 7, we discuss the relationship between these error estimators and prove their equivalence up to higher order terms. We note that the error estimators under consideration are constructed by means of their elementwise contributions according to

$$
\eta=\sum_{T \in \mathcal{T}_{k}} \eta_{T}
$$

Two estimators $\eta^{(1)}$ and $\eta^{(2)}$ are said to be equivalent (locally equivalent), if there exist constants $0<\delta \leq \Delta\left(0<\delta_{T} \leq \Delta_{T}, T \in \mathcal{T}_{k}\right)$, independent of the refinement level, such that

$$
\delta \eta^{(2)} \leq \eta^{(1)} \leq \Delta \eta^{(2)} \quad\left(\delta_{T} \eta_{T}^{(2)} \leq \eta_{T}^{(1)} \leq \Delta_{T} \eta_{T}^{(2)}, T \in \mathcal{T}_{k}\right) .
$$


In view of (1.7), local equivalence implies equivalence but the converse does not necessarily hold true. Neglecting higher order terms, we obtain local equivalence of the residual based error estimator and the hierarchical one. Using this result, we investigate the estimator based on the solution of local subproblems in more detail, and establish local equivalence with the hierarchical estimator in the case of an appropriate modification of the discrete Dirichlet boundary data. As far as the estimator relying on superconvergence results is concerned, we cannot expect equivalence with the other ones. However, adding two additional terms allows us to prove equivalence, whereas no local equivalence can be established.

\section{Definition of the ERror Estimators AND MAin Results}

In the context of standard primal variational problems, a posteriori error estimators are well established. The recent survey articles of Verfürth [27] and Bornemann et al. [8] give an excellent comparison of different kinds of error estimators in the conforming setting. These concepts have been generalized to nonconforming finite element discretizations by Crouzeix-Raviart elements of lowest order in [21, 31]. For mixed finite element methods, there only exists some work of Braess et al. [9], Braess and Verfürth [10] and Verfürth [28] concerning residual based error estimators and indicators. Here, we use the same techniques as in the conforming case, but the investigation of the estimator for the mixed setting is much more complicated and requires some additional tools. In case of the residual based error estimator, we assume that the coefficient matrix $a$ is a piecewise constant diagonal matrix and $b$ is piecewise constant.

It can be easily seen that the total error $\left(\mathbf{j}_{e}, u_{e}\right):=\left(\mathbf{j}-\tilde{\mathbf{j}}_{h}, u-\tilde{u}_{h}\right)$ satisfies the continuous variational problem

$$
\begin{aligned}
a\left(\mathbf{j}-\tilde{\mathbf{j}}_{h}, \mathbf{q}\right)+b\left(\mathbf{q}, u-\tilde{u}_{h}\right) & =r(\mathbf{q}), & & \mathbf{q} \in H(\operatorname{div} ; \Omega), \\
b\left(\mathbf{j}-\tilde{\mathbf{j}}_{h}, v\right)-c\left(u-\tilde{u}_{h}, v\right) & =-\left(f-\Pi_{0} f, v\right)_{0}, & & v \in L^{2}(\Omega),
\end{aligned}
$$

where the residual $r$ is given by $r(\mathbf{q}):=-a\left(\tilde{\mathbf{J}}_{h}, \mathbf{q}\right)-b\left(\mathbf{q}, \tilde{u}_{h}\right), \mathbf{q} \in H(\operatorname{div} ; \Omega)$, and $\Pi_{0} f$ denotes the $L^{2}$-projection of $f$ onto $W_{0}\left(\Omega ; \mathcal{T}_{k}\right)$.

The basic idea behind the construction of a residual based error estimator for the total error $\left\|u-\tilde{u}_{h}\right\|_{0}+\left\|\mathbf{j}-\tilde{\mathbf{j}}_{h}\right\|_{\text {div }}$ is to use a Helmholtz decomposition of $H(\operatorname{div} ; \Omega)$. We obtain an error estimator which can be easily calculated by means of the available finite element approximation $\left(\tilde{\mathbf{J}}_{h}, \tilde{u}_{h}\right)$. In contrast to the hierarchical error estimator, no additional subproblem has to be solved. This is the main advantage of the residual based error estimator $\hat{\eta}_{R}$ which is determined by its local contributions:

$$
\begin{aligned}
\hat{\eta}_{R}^{2} & :=\sum_{T \in \mathcal{T}_{k}} \hat{\eta}_{R ; T}^{2}, \\
\hat{\eta}_{R ; T}^{2} & :=\left\|f-\Pi_{0} f\right\|_{0 ; T}^{2}+h_{T}^{2}\left\|a^{-1} \tilde{\mathbf{j}}_{h}\right\|_{0 ; T}^{2}+\sum_{i=1}^{3} w_{i} \alpha_{e_{i}} h_{e_{i}}\left\|\left[a^{-1} \tilde{\mathbf{j}}_{h} \cdot \mathbf{t}_{e_{i}}\right]_{J}\right\|_{0 ; e_{i}}^{2} .
\end{aligned}
$$

Here, the weighting factors $\alpha_{e_{i}}$ and $w_{i}$ are defined by $\alpha_{e_{i}}:=\frac{1}{4}\left(\alpha_{0}^{T_{1}}+\alpha_{0}^{T_{2}}+\alpha_{1}^{T_{1}}+\alpha_{1}^{T_{2}}\right)$ and $w_{i}:=1 / 2$, if $e_{i}=\partial T_{1} \cap \partial T_{2}$ is an interior edge, and by $\alpha_{e_{i}}:=\frac{1}{2}\left(\alpha_{0}^{T_{e}}+\alpha_{1}^{T_{e}}\right)$ and $w_{i}:=1$, if $e_{i} \subset \partial T_{e} \cap \partial \Omega$. The jump [.] $]_{J}$ on $e$ has to be defined as

$$
\left[a^{-1} \tilde{\mathbf{j}}_{h} \cdot \mathbf{t}_{e}\right]_{J}:=\left(\left.a^{-1} \tilde{\mathbf{j}}_{h} \cdot \mathbf{t}_{e}\right|_{T_{i}}-\left.a^{-1} \tilde{\mathbf{j}}_{h} \cdot \mathbf{t}_{e}\right|_{T_{a}}\right), \quad \partial T_{i} \cap \partial T_{a}=e,
$$




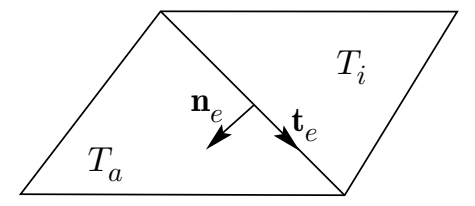

FiguRE 2.1. Orientation of $\mathbf{n}_{e}$ and $\mathbf{t}_{e}$

if $e$ is an interior edge, and

$$
\left[a^{-1} \tilde{\mathbf{j}}_{h} \cdot \mathbf{t}_{e}\right]_{J}:=\left(\left.a^{-1} \tilde{\mathbf{j}}_{h} \cdot \mathbf{t}_{e}\right|_{T_{i}}\right), \quad \partial T_{i} \cap \partial \Omega \supset e,
$$

if $e \subset \partial \Omega$. For the orientation of $\mathbf{t}_{e}$, we refer to Figure 2.1.

This error estimator provides an upper and a lower bound for the total error $\left\|u-\tilde{u}_{h}\right\|_{0}+\left\|\mathbf{j}-\tilde{\mathbf{j}}_{h}\right\|_{\text {div }}$, if the gridsize and the iteration error are small enough.

Theorem 2.1. There exist constants $h_{\max }>0$ and $c_{R}, C_{R}, C_{i t}>0$, independent of the refinement level, such that for all $h \leq h_{\max }$

$$
c_{R} \hat{\eta}_{R}^{2} \leq\left\|u-\tilde{u}_{h}\right\|_{0}^{2}+\left\|\mathbf{j}-\tilde{\boldsymbol{\jmath}}_{h}\right\|_{\text {div }}^{2} \leq C_{R} \hat{\eta}_{R}^{2}+C_{i t}\left(\left\|u_{h}-\tilde{u}_{h}\right\|_{0}^{2}+\left\|\mathbf{j}_{h}-\tilde{\boldsymbol{\jmath}}_{h}\right\|_{\text {div }}^{2}\right) .
$$

In the case of standard conforming finite element discretizations, the hierarchical basis error estimator has been investigated by Deuflhard, Leinen, Yserentant [14]. An excellent overview is given by Bornemann et al. [8]. Recently, this concept has been generalized by Achchab et al. [1] for the mixed setting, but no easily accessible local error estimator is proposed. Here, the introduction of the error estimator is based on the principle of defect correction in higher order ansatz spaces. By means of appropriate localization and decoupling techniques of the flux ansatz space, we obtain an easily computable, efficient and reliable a posteriori error estimator for the flux error and the total error. The hierarchical error estimator presented in this section has been investigated in $[19,20,31]$. We summarize the main ideas and the basic results; for details we refer to the papers cited above.

The hierarchical basis error estimator is based on a discrete defect problem considered on appropriately chosen higher dimensional ansatz spaces. There are two different approaches. Either we consider the same mixed ansatz spaces associated with a finer triangulation, e.g., obtained by uniform refinement from the actual one, or we use higher order mixed ansatz spaces providing improved a priori estimates. Here, we restrict ourselves to the second approach, and consider the Raviart-Thomas ansatz space $R T_{1}\left(\Omega ; \mathcal{T}_{k}\right)$. In contrast to the residual based error estimator, the continuous defect problem (2.1) will not be considered. Instead, to obtain an appropriate approximation of (2.1) we use the higher dimensional Raviart-Thomas ansatz space $R T_{1}\left(\Omega ; \mathcal{T}_{k}\right)$ for the flux and the ansatz space $W_{1}\left(\Omega ; \mathcal{T}_{k}\right)$ of piecewise linear functions for the primal variable. We restrict ourselves to the discrete saddle point problem which requires the computation of a pair $\left(e_{\mathbf{j}}, e_{u}\right) \in R T_{1}\left(\Omega ; \mathcal{T}_{k}\right) \times W_{1}\left(\Omega ; \mathcal{T}_{k}\right)$ such that

$$
\begin{aligned}
a\left(e_{\mathbf{j}}, \mathbf{q}\right)+b\left(\mathbf{q}, e_{u}\right) & =r(\mathbf{q}), & & \mathbf{q} \in R T_{1}\left(\Omega ; \mathcal{T}_{k}\right), \\
b\left(e_{\mathbf{j}}, v\right)-c\left(e_{u}, v\right) & =-\left(f-\Pi_{0} f, v\right)_{0}, & & v \in W_{1}\left(\Omega ; \mathcal{T}_{k}\right) .
\end{aligned}
$$

Denoting by $\left(\mathbf{j}_{R T_{1}}, u_{R T_{1}}\right)$ the solution of the discrete variational problem (1.4) on $R T_{1}\left(\Omega ; \mathcal{T}_{k}\right) \times W_{1}\left(\Omega ; \mathcal{T}_{k}\right)$, the introduction and the analysis of the error estimator 
are based on the following saturation assumptions:

$$
\begin{aligned}
& \left\|\mathbf{j}-\mathbf{j}_{R T_{1}}\right\|_{\text {div }} \leq \beta_{k}\left\|\mathbf{j}-\mathbf{j}_{h}\right\|_{\text {div }}, \quad \beta_{k}>0 \\
& \left\|\mathbf{j}-\mathbf{j}_{R T_{1}}\right\|_{\text {div }}^{2}+\left\|u-u_{R T_{1}}\right\|_{0}^{2} \leq \hat{\beta}_{k}\left(\left\|\mathbf{j}-\mathbf{j}_{h}\right\|_{\text {div }}^{2}+\left\|u-u_{h}\right\|_{0}^{2}\right), \quad \hat{\beta}_{k}>0,
\end{aligned}
$$

with $\beta_{k} \leq \beta_{\infty}<1$ and $\hat{\beta}_{k} \leq \hat{\beta}_{\infty}<1$. These saturation assumptions are motivated by the well known a priori error estimates for $\mathbf{j}-\mathbf{j}_{h}, \mathbf{j}-\mathbf{j}_{R T_{1}}$ and $u-u_{h}, u-u_{R T_{1}}$ (see, e.g., [12]). In particular, the saturation assumption (S1 a) implies both an upper and a lower bound for the total error in terms of the $\|\cdot\|_{\operatorname{div}}$-norms of $e_{\mathbf{j}}$ and the iteration error $\mathbf{j}_{h}-\tilde{\mathbf{j}}_{\mathbf{h}}$ :

$$
\begin{aligned}
& \left(1+\beta_{\infty}\right)^{-1}\left(\left\|e_{\mathbf{j}}\right\|_{\text {div }}-\beta_{\infty}\left\|\mathbf{j}_{h}-\tilde{\mathbf{j}}_{h}\right\|_{\text {div }}\right) \leq\left\|\mathbf{j}-\tilde{\mathbf{j}}_{h}\right\|_{\text {div }}, \\
& \left(1-\beta_{\infty}\right)^{-1}\left(\left\|e_{\mathbf{j}}\right\|_{\text {div }}+\beta_{\infty}\left\|\mathbf{j}_{h}-\tilde{\mathbf{j}}_{h}\right\|_{\text {div }}\right) \geq\left\|\mathbf{j}-\tilde{\mathbf{j}}_{h}\right\|_{\text {div }} .
\end{aligned}
$$

Therefore, only the solution of (2.3) has to be examined. The approximation of $\left(e_{\mathbf{j}}, e_{u}\right)$ is based on the hierarchical two-level splitting of the mixed ansatz spaces $R T_{1}\left(\Omega ; \mathcal{T}_{k}\right)$ and $W_{1}\left(\Omega ; \mathcal{T}_{k}\right)$. By means of the $L^{2}$-projection $\Pi$ of $W_{1}\left(\Omega ; \mathcal{T}_{k}\right)$ onto $W_{0}\left(\Omega ; \mathcal{T}_{k}\right)$ and the interpolation operator $\rho: R T_{1}\left(\Omega ; \mathcal{T}_{k}\right) \longrightarrow R T_{0}\left(\Omega ; \mathcal{T}_{k}\right)$ given locally by

$$
\int_{e} \mathbf{n}_{e} \cdot(\rho \mathbf{q}) d \sigma=\int_{e} \mathbf{n}_{e} \cdot \mathbf{q} d \sigma, \quad e \in \mathcal{E}_{k},
$$

we obtain a hierarchical splitting of $W_{1}\left(\Omega ; \mathcal{T}_{k}\right)$ and $R T_{1}\left(\Omega ; \mathcal{T}_{k}\right)$ according to

$$
\begin{gathered}
W_{1}\left(\Omega ; \mathcal{T}_{k}\right)=W_{0}\left(\Omega ; \mathcal{T}_{k}\right) \oplus \widehat{W}_{1}\left(\Omega ; \mathcal{T}_{k}\right), \\
R T_{1}\left(\Omega ; \mathcal{T}_{k}\right)=R T_{0}\left(\Omega ; \mathcal{T}_{k}\right) \oplus \widehat{R T}_{1}\left(\Omega ; \mathcal{T}_{k}\right),
\end{gathered}
$$

where $\widehat{W}_{1}\left(\Omega ; \mathcal{T}_{k}\right):=(\operatorname{Id}-\Pi) W_{1}\left(\Omega ; \mathcal{T}_{k}\right)$ and $\widehat{R T}_{1}\left(\Omega ; \mathcal{T}_{k}\right):=(\operatorname{Id}-\rho) R T_{1}\left(\Omega ; \mathcal{T}_{k}\right)$. The hierarchical surplus in the flux, $\widehat{R T}_{1}\left(\Omega ; \mathcal{T}_{k}\right)$, can be further decomposed into a divergence free part $\widehat{R T}_{1}^{0}\left(\Omega ; \mathcal{T}_{k}\right)$ and its complement $\widehat{R T}_{1}^{1}\left(\Omega ; \mathcal{T}_{k}\right)$ :

$$
\widehat{R T}_{1}\left(\Omega ; \mathcal{T}_{k}\right)=\widehat{R T}_{1}^{0}\left(\Omega ; \mathcal{T}_{k}\right) \oplus \widehat{R T}_{1}^{1}\left(\Omega ; \mathcal{T}_{k}\right)
$$

where

$$
\begin{aligned}
& \widehat{R T}_{1}^{0}\left(\Omega ; \mathcal{T}_{k}\right):=\operatorname{curl} \hat{S}_{2}\left(\Omega ; \mathcal{T}_{k}\right) \\
& \widehat{R T}_{1}^{1}\left(\Omega ; \mathcal{T}_{k}\right):=\left\{\mathbf{q} \in \widehat{R T}_{1}\left(\Omega ; \mathcal{T}_{k}\right)\left|\mathbf{n}_{e} \cdot \mathbf{q}\right|_{e}=0, e \in \mathcal{E}_{k}\right\}
\end{aligned}
$$

Here, $\hat{S}_{2}\left(\Omega ; \mathcal{T}_{k}\right)$ stands for the space of quadratic bubble functions associated with the midpoints of the edges. This space is given in terms of the hierarchical twolevel splitting $S_{2}\left(\Omega ; \mathcal{T}_{k}\right)=S_{1}\left(\Omega ; \mathcal{T}_{k}\right) \oplus \hat{S}_{2}\left(\Omega ; \mathcal{T}_{k}\right)$, where $S_{1}\left(\Omega ; \mathcal{T}_{k}\right)$ and $S_{2}\left(\Omega ; \mathcal{T}_{k}\right)$ refer to the conforming ansatz spaces associated with the standard P1 and P2 approximations. The structure of the decomposition (2.6) and (2.7) is symbolized in Figure 2.2. The splitting (2.7) is somewhat similar to the Helmholtz decomposition of the $H(\operatorname{div} ; \Omega)$ ansatz space that will be used in case of the residual based error estimator (see Section 3 below). But in contrast to this Helmholtz decomposition, the ansatz spaces $\widehat{R T}_{1}^{0}\left(\Omega ; \mathcal{T}_{k}\right)$ and $\widehat{R T}_{1}^{1}\left(\Omega ; \mathcal{T}_{k}\right)$ are not orthogonal with respect to the bilinear form $a(\cdot, \cdot)$. Furthermore, it is easy to see that $\widehat{R T}_{1}^{1}\left(\Omega ; \mathcal{T}_{k}\right)$ can be written 


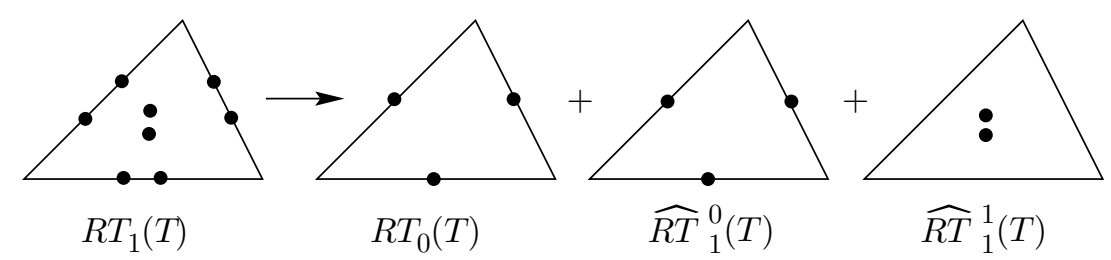

FiguRE 2.2. Degrees of freedom of the subspaces of the higher order Raviart-Thomas space

as the direct sum of local two-dimensional subspaces $\widehat{R T}_{1}^{1}(T)$ which correspond to the "interior" degrees of freedom of $R T_{1}(T)$ :

$$
\widehat{R T}_{1}^{1}\left(\Omega ; \mathcal{T}_{k}\right)=\bigoplus_{T \in \mathcal{T}_{k}} \widehat{R T}_{1}^{1}(T), \widehat{R T}_{1}^{1}(T):=\left\{\mathbf{q} \in \widehat{R T}_{1}^{1}\left(\Omega ; \mathcal{T}_{k}\right)|\mathbf{q}|_{\Omega \backslash T}=0\right\} .
$$

Due to the special structure of $W_{1}\left(\Omega ; \mathcal{T}_{k}\right)$, the subspace $\widehat{W}_{1}\left(\Omega ; \mathcal{T}_{k}\right)$ can be decomposed into the direct sum of local two-dimensional subspaces:

$$
\widehat{W}_{1}\left(\Omega ; \mathcal{T}_{k}\right)=\bigoplus_{T \in \mathcal{T}_{k}} \widehat{W}_{1}(T), \quad \widehat{W}_{1}(T):=\left\{v \in \widehat{W}_{1}\left(\Omega ; \mathcal{T}_{k}\right)|v|_{\Omega \backslash T}=0\right\} .
$$

For the definition of the error estimator we have to consider two different types of local low dimensional variational problems associated with the two parts $\widehat{R T}_{1}^{0}\left(\Omega ; \mathcal{T}_{k}\right)$ and $\widehat{R T}_{1}^{1}\left(\Omega ; \mathcal{T}_{k}\right)$ of the hierarchical surplus. For each edge of the triangulation we have to solve a single equation

$$
a\left(\operatorname{curl} \varphi_{e}, \operatorname{curl} \Phi_{e}\right)=r\left(\operatorname{curl} \Phi_{e}\right),
$$

where $\Phi_{e}$ is the quadratic bubble function associated with the edge $e$ and $\varphi_{e} \in$ $\operatorname{span}\left\{\Phi_{e}\right\}$. The second variational problem is associated with the elements $T$. For each element we have to consider a $4 \times 4$ saddle point problem: Find $\left(\tilde{e}_{\mathbf{j}_{1}}^{1}, \tilde{e}_{u_{1}}\right) \in$ $\widehat{R T}_{1}^{1}(T) \times \widehat{W}_{1}(T)$ such that

$$
\begin{aligned}
\left.a\right|_{T}\left(\tilde{e}_{\mathbf{j}_{1}}^{1}, \mathbf{q}\right)+\left.b\right|_{T}\left(\mathbf{q}, \tilde{e}_{u_{1}}\right) & =\left.r\right|_{T}(\mathbf{q}), & & \mathbf{q} \in \widehat{R T}_{1}^{1}(T), \\
\left.b\right|_{T}\left(\tilde{e}_{\mathbf{j}_{1}}^{1}, v\right)-\left.c\right|_{T}\left(\tilde{e}_{u_{1}}, v\right) & =(f, v)_{0 ; T}, & & v \in \widehat{W}_{1}(T) .
\end{aligned}
$$

The solutions of (2.8) and (2.9) lead to the local definition of an a posteriori fluxoriented error estimator $\eta_{H}$ :

$$
\begin{aligned}
\eta_{H}^{2} & :=\sum_{T \in \mathcal{T}_{k}} \eta_{H ; T}^{2}, \\
\eta_{H ; T}^{2} & :=\left\|\tilde{e}_{\mathbf{j}_{1}}^{1}\right\|_{\text {div } ; T}^{2}+\sum_{i=1}^{3} w_{i}\left\|\operatorname{curl} \varphi_{e_{i}}\right\|_{\text {div }}^{2}, \quad T \in \mathcal{T}_{k},
\end{aligned}
$$

where the weighting factor $w_{i}$ is defined as in the case of the residual based error estimator.

Theorem 2.2. Under the saturation assumption (S1 a) with $0<\beta_{k}<\beta_{\infty}<$ 1 there exist constants $c_{\text {hier }}, C_{\text {hier }}>0$ and $\gamma_{\text {hier }}, \Gamma_{\text {hier }}>0$, independent of the 
refinement level, such that

$$
\begin{gathered}
\left(1+\beta_{\infty}\right)^{-1}\left(c_{\text {hier }} \eta_{H}-\gamma_{h i e r}\left\|\mathbf{j}_{h}-\tilde{\boldsymbol{\jmath}}_{h}\right\|_{\text {div }}\right) \leq\left\|\mathbf{j}-\tilde{\boldsymbol{\jmath}}_{h}\right\|_{\text {div }}, \\
\left(1-\beta_{\infty}\right)^{-1}\left(C_{\text {hier }} \eta_{H}+\Gamma_{h i e r}\left\|\mathbf{j}_{h}-\tilde{\boldsymbol{\jmath}}_{h}\right\|_{\text {div }}\right) \geq\left\|\mathbf{j}-\tilde{\boldsymbol{\jmath}}_{h}\right\|_{\text {div }} .
\end{gathered}
$$

Next, we consider an error estimator $\eta_{L}$ based on the solution of local subproblems that is strongly related to the hierarchical error estimator. In the standard conforming setting, this kind of error estimator is due to Bank and Weiser [6]. For nonconforming techniques we refer to [21]. It relies on a defect correction with respect to a higher order ansatz space and an appropriate localization based on a hierarchical two-level splitting. It turns out that the estimator can be computed elementwise by the solution of local Dirichlet problems similar to the original global one (cf. [21]). In contrast to the standard conforming and nonconforming setting, the boundary data cannot be obtained by a simple averaging of the computed approximation.

For simplicity, we only consider the discretization error $\left(\mathbf{j}-\mathbf{j}_{h}, u-u_{h}\right)$ and not the total error $\left(\mathbf{j}-\tilde{\mathbf{j}}_{h}, u-\tilde{u}_{h}\right)$. Note that the results can be generalized to the total error. This time, we state the defect problem as a local Dirichlet problem for each element $T \in \mathcal{T}_{k}$ :

$$
\begin{aligned}
\left.a\right|_{T}\left(\mathbf{j}-\mathbf{j}_{h}, \mathbf{q}\right)+\left.b\right|_{T}\left(\mathbf{q}, u-u_{h}\right) & =-\int_{\partial T} u \mathbf{n} \cdot \mathbf{q} d \sigma+\left.r\right|_{T}(\mathbf{q}), & & \mathbf{q} \in H(\operatorname{div} ; T), \\
\left.b\right|_{T}\left(\mathbf{j}-\mathbf{j}_{h}, v\right)-\left.c\right|_{T}\left(u-u_{h}, v\right) & =-\left(f-\Pi_{0} f, v\right)_{0 ; T}, & & v \in L^{2}(T) .
\end{aligned}
$$

As in the case of the hierarchical error estimator, we only look for an adequate approximation of the solution of (2.12). The original ansatz space $H(\operatorname{div} ; T) \times L^{2}(T)$ will be replaced by $R T_{1}(T) \times P_{1}(T)$, and the Dirichlet data $u$ by some appropriate approximation $u_{D}$ which can be easily calculated from $\left(\mathbf{j}_{h}, u_{h}\right)$. A possible choice of $u_{D}$ will be discussed in Section 5 . In contrast to the hierarchical error estimator, we need an additional saturation assumption concerning the approximation of the Dirichlet data. We refer to $R T_{1}^{-1}\left(\Omega ; \mathcal{T}_{k}\right)$ as the nonconforming ansatz space, where

$$
R T_{l}^{-1}\left(\Omega ; \mathcal{T}_{k}\right):=\left\{\mathbf{q} \in\left(L^{2}(\Omega)\right)^{2}|\mathbf{q}|_{T} \in R T_{l}(T), \quad T \in \mathcal{T}_{k}\right\}, \quad 0 \leq l \leq 1,
$$

and to $\lambda_{R T_{1}}$ as the Lagrange multiplier which is a piecewise linear function on the edges uniquely determined by means of $\mathbf{q} \in R T_{1}^{-1}\left(\Omega ; \mathcal{T}_{k}\right)$,

$$
\sum_{T \in \mathcal{T}_{k}} \int_{\partial T} \lambda_{R T_{1}} \mathbf{n} \cdot \mathbf{q} d \sigma=-\sum_{T \in \mathcal{T}_{k}} \int_{T} a^{-1} \mathbf{j}_{R T_{1}} \cdot \mathbf{q} d x+\sum_{T \in \mathcal{T}_{k}} \int_{T} \operatorname{div} \mathbf{q} u_{R T_{1}} d x .
$$

Then, introducing the weighted norm $\|\cdot\|_{0 ; \hat{\mathcal{E}}_{k}^{-1}}$ according to

$$
\|v\|_{0 ; \hat{\mathcal{E}}_{k}^{-1}}:=\left(\sum_{e \in \mathcal{E}_{k}} h_{e}^{-1} \alpha_{e}\|v\|_{0 ; e}^{2}\right)^{1 / 2}, \quad v \in L^{2}\left(\mathcal{E}_{k}\right)
$$

we assume

$$
\left\|\lambda_{R T_{1}}-\left[u_{D}\right]_{A}\right\|_{0 ; \hat{\mathcal{E}}_{k}^{-1}} \leq \beta_{2 ; k}\left\|\mathbf{j}-\mathbf{j}_{h}\right\|_{\text {div }}, \quad \beta_{2 ; k}>0,
$$

to hold with $\beta_{2 ; k} \leq \beta_{2 ; \infty}<\infty$, where the average $[v]_{A}$ on $e, v \in \prod_{T \in \mathcal{T}_{k}} H^{1}(T)$, is given by $[v]_{A}:=\frac{1}{2}\left(\left.v\right|_{T_{i}}+\left.v\right|_{T_{a}}\right), \partial T_{i} \cap \partial T_{a}=e$, if $e$ is an interior edge, and by $[v]_{A}:=\frac{1}{2}\left(\left.v\right|_{T_{i}}\right), \partial T_{i} \cap \partial \Omega \supset e$, if $e \subset \partial \Omega$. In general, assumption (S2) is 
motivated by an adequate a priori error estimate or by some equivalence results (see subsection 7.2). Because of the saturation assumptions (S1 a) and (S2), the previous simplifications are justified. We consider the discretized defect problem

$$
\begin{aligned}
\left.a\right|_{T}\left(\left.\hat{e}_{\mathbf{j}}\right|_{T}, \mathbf{q}\right)+\left.b\right|_{T}\left(\mathbf{q},\left.\hat{e}_{u}\right|_{T}\right) & =-\int_{\partial T}\left[u_{D}\right]_{A} \mathbf{n} \cdot \mathbf{q} d \sigma+\left.r\right|_{T}(\mathbf{q}), & & \mathbf{q} \in R T_{1}(T) \\
\left.b\right|_{T}\left(\left.\hat{e}_{\mathbf{j}}\right|_{T}, v\right)-\left.c\right|_{T}\left(\left.\hat{e}_{u}\right|_{T}, v\right) & =-\left(f-\Pi_{0}, v\right)_{0 ; T}, & & v \in P_{1}(T)
\end{aligned}
$$

Then $\hat{e}_{\mathbf{j}}:=\left(\left.\hat{e}_{\mathbf{j}}\right|_{T}\right)_{T \in \mathcal{T}_{k}}$ does guarantee lower and upper bounds for the error in the flux.

Theorem 2.3. Under the saturation assumptions (S1 a) with $0<\beta_{k}<\beta_{\infty}<1$ and (S2) with $0<\beta_{2 ; k}<\beta_{2 ; \infty}<\infty$ there exist constants $c_{l o c}, C_{l o c}>0$, independent of the refinement level, such that

$$
c_{l o c}\left\|\hat{e}_{\mathbf{j}}\right\|_{\text {div }} \leq\left\|\mathbf{j}-\mathbf{j}_{h}\right\|_{\text {div }} \leq\left(1-\beta_{\infty}\right)^{-1} C_{l o c}\left\|\hat{e}_{\mathbf{j}}\right\|_{\text {div }} .
$$

Finally, we use the same hierarchical splittings and decoupling techniques as before. For each element we thus obtain three scalar equations and one saddle point problem that have to be solved. The error estimator $\eta_{L}$ is defined by

$$
\begin{aligned}
\eta_{L}^{2} & :=\sum_{T \in \mathcal{T}_{k}} \eta_{L ; T}^{2}, \\
\eta_{L ; T}^{2} & :=\left\|\hat{e}_{\mathbf{j}_{1}}^{1}\right\|_{\text {div } ; T}^{2}+\left.\sum_{i=1}^{3} a\right|_{T}\left(\operatorname{curl} \varphi_{e_{i} ; T}, \operatorname{curl} \varphi_{e_{i} ; T}\right), \quad T \in \mathcal{T}_{k},
\end{aligned}
$$

where $\hat{e}_{\mathbf{j}_{1}}^{1}$ and $\operatorname{curl} \varphi_{e_{i} ; T}$ are the solutions of the local problems on the element $T$.

Theorem 2.4. Let the saturation assumptions (S1 a) with $\beta_{\infty}<1$ and (S2) with $\beta_{2 ; \infty}<\infty$ be satisfied. Then, there exist constants $0<c_{l o c} \leq C_{l o c}$, independent of the refinement level, such that

$$
c_{l o c} \eta_{L} \leq\left\|\mathbf{j}-\mathbf{j}_{h}\right\|_{\text {div }} \leq C_{l o c} \eta_{L} .
$$

In Section 5, we will show that $\hat{e}_{\mathbf{j}_{1}}^{1}=\tilde{e}_{\mathbf{j}_{1}}^{1}$, and thus the first part of the error estimator is exactly the same as in the case of the hierarchical basis error estimator.

Finally, in Section 6 we propose an estimator $\eta_{S}$ for the error in the primal variable that can be motivated by a superconvergence result for the technique of mixed hybridization. In contrast to the hierarchical basis error estimator $\eta_{H}$ and the error estimator $\eta_{L}$ based on the solution of local subproblems, we do not have to solve additional defect problems. In the standard conforming case, error estimators obtained by some postprocessing of the approximation have been introduced by Zienkiewicz and Zhu in $[32,34]$ and have been further analyzed by Rodriguez [24]. In the mixed setting there is some work of Brandts [11]. In contrast to the conforming situation, we will be able to prove the equivalence between $\eta_{S}$ and the $L^{2}$-norm of the difference of $\tilde{u}_{h}$ and some higher order finite element approximation which turns out to be the solution of a modified nonconforming variational approach. Therefore, we have a strong relationship between the error estimator and a discrete defect problem which is solved in a higher order ansatz space. Details will be given 
in Section 6. The error estimator $\eta_{S}$ is given as follows:

$$
\begin{aligned}
\eta_{S}^{2} & :=\sum_{T \in \mathcal{T}_{k}} \eta_{S ; T}^{2}, \\
\eta_{S ; T}^{2} & :=\sum_{i=1}^{3} w_{i} h_{e_{i}}^{2}\left(\left.\left[\tilde{u}_{h}\right]_{J}\right|_{e_{i}}\right)^{2},
\end{aligned}
$$

where the weighting factor $w_{i}$ is defined as before.

Theorem 2.5. Let $\tilde{u}_{h} \in W_{0}\left(\Omega ; \mathcal{T}_{k}\right)$ be an approximation of the primal variable $u$ obtained by an iterative solution process for system (1.4). Then, there exist constants $0<\sigma_{0} \leq \sigma_{1}$ and $0<C_{0}<C_{1}$, depending only on the shape regularity of $\mathcal{T}_{k}$ and the ellipticity constants in (1.2), such that

$$
\sigma_{0} \eta_{S}-C_{0}\left\|u_{h}-\tilde{u}_{h}\right\|_{0} \leq\left\|u-\tilde{u}_{h}\right\|_{0} \leq \sigma_{1} \eta_{S}+C_{1}\left\|u_{h}-\tilde{u}_{h}\right\|_{0} .
$$

We emphasize that the computation of the estimator only involves the jumps of the piecewise constant approximation for the primal variable across the inner element boundaries.

In [11], Brandts introduced an a posteriori error estimator for the flux in the $L^{2}$-norm based on a higher order recovering of the flux. If we add this estimator and $\left\|f-\Pi_{0} f\right\|_{0}$ to $\eta_{S}$, it turns out that we again obtain an error estimator $\hat{\eta}_{S}$ for the total error.

The error estimator in [11] is defined as

$$
\left\|\mathbf{j}_{h}-K \mathbf{j}_{h}\right\|_{0}
$$

where the linear operator $K: R T_{0}\left(\Omega ; \mathcal{T}_{k}\right) \longrightarrow\left(C R\left(\Omega ; \mathcal{T}_{k}\right)\right)^{2}$ is locally given by its value at the midpoint $m_{e}$ of the edges of the triangulation

$$
K \mathbf{p}\left(m_{e}\right):=\frac{1}{2}\left(\left.\mathbf{p}\right|_{T_{1}}+\left.\mathbf{p}\right|_{T_{2}}\right)\left(m_{e}\right), \quad T_{1} \cap T_{2}=e \in \mathcal{E}_{k},
$$

if $e$ is an interior edge, and by

$$
\mathbf{t} \cdot(K \mathbf{p})\left(m_{e}\right):=0, \quad \mathbf{n} \cdot(K \mathbf{p})\left(m_{e}\right):=\left.\mathbf{n} \cdot \mathbf{p}\right|_{T_{e}}, \quad e \subset \partial T_{e},
$$

if $e$ is an edge on the boundary $\partial \Omega$.

The error estimator $\hat{\eta}_{s}$ is then given by

$$
\begin{aligned}
\hat{\eta}_{S}^{2} & :=\sum_{T \in \mathcal{T}_{k}} \hat{\eta}_{S ; T}^{2}, \\
\hat{\eta}_{S ; T}^{2} & :=\eta_{S ; T}^{2}+\left\|\mathbf{j}_{h}-K \mathbf{j}_{h}\right\|_{0 ; T}^{2}+\left\|f-\Pi_{0} f\right\|_{0 ; T}^{2} .
\end{aligned}
$$

In subsection 7.3 , it will be shown that $\hat{\eta}_{S}$ and the residual based error estimator $\hat{\eta}_{R}$ are globally equivalent.

\section{The RESIDUAL BASED ERROR ESTIMATOR}

First, we summarize some technical results which are an indispensable tool for the investigation of the residual based error estimator. We consider the projection operator $P_{C}: L^{2}(\Omega) \rightarrow S_{1}\left(\Omega ; \mathcal{T}_{k}\right)$ with respect to a discrete $L^{2}$-norm due to Clément [13]. In contrast to the Lagrangian interpolation operator, this operator can be applied to discontinuous functions. Denoting by $\tilde{D}_{p}:=\bigcup\left\{T \in \mathcal{T}_{k} \mid p \in \partial T\right\}$ the union of all triangles containing the vertex $p$ and by $\lambda_{p}$ the linear conforming nodal basis function, i.e., $\lambda_{p}\left(p^{\prime}\right)=\delta_{p, p^{\prime}}$, it is defined by

$$
P_{C} v:=\sum_{p \in \mathcal{P}_{k}}\left(q_{p}(v)\right)(p) \lambda_{p}, \quad v \in L^{2}(\Omega),
$$


where $q_{p}(v) \in P_{1}\left(\tilde{D}_{p}\right), p \in \mathcal{P}_{k}$, is the projection of $v$ onto the space $P_{1}\left(\tilde{D}_{p}\right)$,

$$
\left(q_{p}, q\right)_{0 ; \tilde{D}_{p}}=(v, q)_{0 ; \tilde{D}_{p}}, \quad q \in P_{1}\left(\tilde{D}_{p}\right) .
$$

$P_{C}$ has the following properties:

Lemma 3.1. Let $v \in H^{1}(\Omega), T \in \mathcal{T}_{k}$ and $e \in \mathcal{E}_{k}, k \geq 0$. Then, there exist constants $C_{0 ; C}, C_{1 ; C}, \hat{C}_{0 ; C}>0$, independent of the refinement level, such that

$$
\begin{gathered}
\left\|v-P_{C} v\right\|_{0 ; T} \leq h_{T} C_{0 ; C}|v|_{1 ; \tilde{D}_{T}}, \\
\left\|\hat{a}^{1 / 2} \nabla P_{C} v\right\|_{0 ; T} \leq C_{1 ; C}\left\|\hat{a}^{1 / 2} \nabla v\right\|_{0 ; \tilde{D}_{T}}, \\
\hat{a}=\left(\hat{a}_{i j}\right)_{i, j=1}^{2}, \hat{a}_{11}:=\left(a^{-1}\right)_{22}, \hat{a}_{22}:=\left(a^{-1}\right)_{11}, \hat{a}_{12}=\hat{a}_{21}:=0, \\
\left\|v-P_{C} v\right\|_{0 ; e} \leq \sqrt{\alpha_{e} h_{e}} \hat{C}_{0 ; C}\left\|\hat{a}^{-1 / 2} \nabla v\right\|_{0 ; \tilde{D}_{e}}
\end{gathered}
$$

where $\tilde{D}_{e}:=\bigcup\left\{T \in \mathcal{T}_{k} \mid e \cap \partial T \neq \emptyset\right\}, \tilde{D}_{T}:=\bigcup\left\{T^{\prime} \in \mathcal{T}_{k} \mid \partial T^{\prime} \cap \partial T \neq \emptyset\right\}$, $\alpha_{e}:=\frac{1}{4}\left(\alpha_{0}^{T_{1}}+\alpha_{0}^{T_{2}}+\alpha_{1}^{T_{1}}+\alpha_{1}^{T_{2}}\right), \partial T_{1} \cap \partial T_{2}=e \subset \Omega$ and $\alpha_{e}:=\frac{1}{2}\left(\alpha_{0}^{T_{e}}+\alpha_{1}^{T_{e}}\right)$, $e \subset \partial T_{e} \cap \partial \Omega$.

For the proof we refer to [13] and remark that the matrix $\hat{a}$ is strictly positive definite.

We shall also take advantage of the following approximation property of the $L^{2}$-projection $\Pi_{0}$ onto the space of piecewise constant functions:

$$
\left\|v-\Pi_{0} v\right\|_{0 ; T} \leq C_{\text {proj }} h_{T}|v|_{1 ; T}, \quad v \in H^{1}(T), T \in \mathcal{T}_{k},
$$

where $C_{\text {proj }}$ stands for a positive constant independent of the refinement level.

We further need two different types of bubble functions $\Phi_{T}, T \in \mathcal{T}_{k}$, and $\Phi_{e}$, $e \in \mathcal{E}_{k}$, associated with the element $T$ and the edge $e$, respectively. Denoting by $\lambda_{p_{i}}^{T}$, $p_{i} \in \mathcal{P}_{k} \cap \partial T, 1 \leq i \leq 3$, the barycentric coordinates of $T \in \mathcal{T}_{k}$ and by $p_{i}^{e} \in \mathcal{P}_{k} \cap e$, $1 \leq i \leq 2$, the vertices of the edge $e \subset \partial T$, they are defined as follows:

$$
\Phi_{T}:=27 \lambda_{p_{1}}^{T} \lambda_{p_{2}}^{T} \lambda_{p_{3}}^{T}, \quad \Phi_{e}:=4 \lambda_{p_{1}^{\mathrm{e}}}^{T} \lambda_{p_{2}^{\mathrm{e}}}^{T} .
$$

Lemma 3.2. (i) Let $\mathbf{p} \in\left(P_{1}(T)\right)^{2}, T \in \mathcal{T}_{k}, k \geq 0$. Then, there exist constants $C_{0 ; D}, C_{1 ; D}>0$ independent of $T \in \mathcal{T}_{k}$ such that

$$
\begin{gathered}
\|\mathbf{p}\|_{0 ; T}^{2} \leq C_{0 ; D}^{2} \int_{T} \mathbf{p} \cdot \Phi_{T} \mathbf{p} d x, \\
\left\|\operatorname{div}\left(\Phi_{T} \mathbf{p}\right)\right\|_{0 ; T} \leq h_{T}{ }^{-1} C_{1 ; D}\|\mathbf{p}\|_{0 ; T} .
\end{gathered}
$$

(ii) Let $p \in P_{1}(e), e \in \mathcal{E}_{k}, k \geq 0$. Then, there exist constants $C_{0 ; K}, C_{1 ; K}>0$, independent of $e \in \mathcal{E}_{k}$, such that

$$
\begin{gathered}
\|p\|_{0 ; e}^{2} \leq C_{0 ; K}^{2} \int_{e} p^{2} \Phi_{e} d \sigma, \\
\left\|\hat{a}^{-1 / 2} \nabla\left(p^{D_{e}} \Phi_{e}\right)\right\|_{0 ; D_{e}} \leq\left(\alpha_{e} h_{e}\right)^{-1 / 2} C_{1 ; K}\|p\|_{0 ; e}, \\
D_{e}:=\bigcup\left\{T \in \mathcal{T}_{k} \mid e \subset \partial T\right\},
\end{gathered}
$$

where $p^{D_{e}}$ defines a prolongation of $p$ on $\Omega, e_{T} \subset \partial T$ fixed:

$$
p^{D_{e}}(x):=\left\{\begin{array}{cl}
0, & x \notin D_{e}, \\
p\left(x_{e}\right), & x \in T \subset D_{e} \text { and } x_{e} \in e,\left(x-x_{e}\right) \| e_{T} \neq e .
\end{array}\right.
$$


The proof is an easy consequence of the affine equivalence of the elements and the inverse inequality for polynomials. Note that the constants depend only on the initial triangulation and on the ratio of the local upper and lower bounds of $a$. The same type of result is used in [27].

The investigation of the a posteriori error estimator will be provided in several steps. In a first step, we decompose the flux ansatz space $H(\operatorname{div} ; \Omega)$ into a solenoidal and a weakly irrotational part:

$$
H(\operatorname{div} ; \Omega)=H^{0}(\operatorname{div} ; \Omega) \oplus H^{1}(\operatorname{div} ; \Omega),
$$

where $H^{0}(\operatorname{div} ; \Omega):=\{\mathbf{q} \in H(\operatorname{div} ; \Omega) \mid \operatorname{div} \mathbf{q}=0\}$ and the orthogonal complement is defined by $H^{1}(\operatorname{div} ; \Omega):=\left\{\mathbf{q} \in H(\operatorname{div} ; \Omega) \mid a\left(\mathbf{q}, \mathbf{q}^{0}\right)=0, \quad \mathbf{q}^{0} \in H^{0}(\operatorname{div} ; \Omega)\right\}$. According to this splitting, the flux error can be written as $\mathbf{j}_{e}=\mathbf{j}_{e}^{0}+\mathbf{j}_{e}^{1}$, where $\mathbf{j}_{e}^{0} \in H^{0}(\operatorname{div} ; \Omega)$ and $\mathbf{j}_{e}^{1} \in H^{1}(\operatorname{div} ; \Omega)$. Note that this representation is unique. Now, we study the subspaces $H^{0}(\operatorname{div} ; \Omega)$ and $H^{1}(\operatorname{div} ; \Omega)$ in more detail.

Lemma 3.3. The following properties of $H^{0}(\operatorname{div} ; \Omega)$ and $H^{1}(\operatorname{div} ; \Omega)$ hold true:

(i) For each element $\mathbf{q} \in H^{0}(\operatorname{div} ; \Omega)$ there exists a scalar function $\phi \in H^{1}(\Omega)$ such that

$$
\mathbf{q}=\operatorname{curl} \phi:=\left(\begin{array}{c}
\phi_{y} \\
-\phi_{x}
\end{array}\right)
$$

(ii) On $H^{1}(\operatorname{div} ; \Omega)$ the following norm equivalence holds true:

$$
c_{\text {div }}\|\operatorname{div} \mathbf{q}\|_{0} \leq\|\mathbf{q}\|_{\text {div }} \leq C_{\text {div }}\|\operatorname{div} \mathbf{q}\|_{0}
$$

with constants $0<c_{\mathrm{div}}<C_{\mathrm{div}}$ independent of $\mathbf{q} \in H^{1}(\operatorname{div} ; \Omega)$.

Proof. The proof of the first assertion can be found in [18, Thm. 3.4], whereas the second assertion is an easy consequence of [12, Prop. 1.2].

The construction of the a posteriori error estimator is mainly based on the preceding splitting of the flux ansatz space. The variational problem (2.1) consists of two independent subproblems. The first subproblem is associated with the solenoidal subspace and gives rise to a positive definite problem:

$$
a\left(\mathbf{j}_{e}^{0}, \mathbf{q}\right)=r(\mathbf{q}), \quad \mathbf{q} \in H^{0}(\operatorname{div} ; \Omega) .
$$

Introducing the weighted norm $\|\cdot\|_{0 ; \mathcal{E}_{k}}$ according to

$$
\|v\|_{0 ; \mathcal{E}_{k}}^{2}:=\sum_{e \in \mathcal{E}_{k}} \alpha_{e} h_{e}\|v\|_{0 ; e}^{2}, \quad v \in L^{2}\left(\mathcal{E}_{k}\right),
$$

we will show that $\left\|\left[a^{-1} \tilde{\mathbf{j}}_{h} \mathbf{t}_{e}\right]_{J}\right\|_{0 ; \mathcal{E}_{k}}$ yields sharp upper and lower bounds for the solenoidal part of the flux error, provided the iteration error is small enough. The existence of a nonvanishing Helmholtz term and the indefiniteness of the saddle point problem (2.1) do not influence the construction of the bounds.

Lemma 3.4. There exist constants $c_{\mathbf{j}_{e}^{0}}, C_{\mathbf{j}_{e}^{0}}, C_{i t}^{0}>0$, independent of the refinement level, such that

$$
\begin{aligned}
& c_{\mathbf{j}_{e}^{0}}\left\|\left[a^{-1} \tilde{\boldsymbol{\jmath}}_{h} \cdot \mathbf{t}_{e}\right]_{J}\right\|_{0 ; \mathcal{E}_{k}} \leq\left\|\mathbf{j}_{e}^{0}\right\|_{\mathrm{div}} \leq C_{\mathbf{j}_{e}^{0}}\left\|\left[a^{-1} \tilde{\boldsymbol{\jmath}}_{h} \cdot \mathbf{t}_{e}\right]_{J}\right\|_{0 ; \mathcal{E}_{k}}+C_{i t}^{0}\left\|\mathbf{j}_{h}-\tilde{\boldsymbol{\jmath}}_{h}\right\|_{\mathrm{div}}, \\
& \text { where } c_{\mathbf{j}_{e}^{0}}:=\left(\sqrt{3} C_{0 ; K}^{2} C_{1 ; K}\right)^{-1}, C_{\mathbf{j}_{e}^{0}}:=\sqrt{\kappa_{K}} \hat{C}_{0 ; C} \text { and } C_{i t}^{0}:=\sqrt{\kappa_{D}} C_{1 ; C} .
\end{aligned}
$$


Proof. Following the same lines as in the conforming setting [27], we evaluate the residual as a continuous linear functional restricted to $H^{0}(\operatorname{div} ; \Omega)$. Let $\mathbf{q}=\operatorname{curl} \phi \in$ $H^{0}(\operatorname{div} ; \Omega)$; then

$$
\begin{aligned}
r(\mathbf{q})= & -a\left(\tilde{\mathbf{J}}_{h}, \mathbf{q}\right)=-\int_{\Omega} a^{-1} \tilde{\mathbf{j}}_{h} \cdot \mathbf{q} d x=-\int_{\Omega} a^{-1} \tilde{\mathbf{j}}_{h} \cdot \operatorname{curl} \phi d x \\
= & \sum_{T \in \mathcal{T}_{k}} \int_{T}\left(a_{1,1}^{-1}\left(\tilde{\mathbf{J}}_{h}\right)_{1, y}-a_{2,2}^{-1}\left(\tilde{\mathbf{J}}_{h}\right)_{2, x}\right) \phi d x \\
& -\sum_{T \in \mathcal{T}_{k}} \int_{\partial T}\left(a_{1,1}^{-1}\left(\tilde{\mathbf{j}}_{h}\right)_{1} \mathbf{n}_{y}-a_{2,2}^{-1}\left(\tilde{\mathbf{J}}_{h}\right)_{2} \mathbf{n}_{x}\right) \phi d \sigma \\
= & \sum_{T \in \mathcal{T}_{k}} \int_{\partial T}\left(a^{-1} \tilde{\mathbf{j}}_{h} \cdot \mathbf{t}\right) \phi d \sigma=\sum_{e \in \mathcal{E}_{k}} \int_{e}\left(\left[a^{-1} \tilde{\mathbf{j}}_{h} \cdot \mathbf{t}_{e}\right]_{J}\right) \phi d \sigma .
\end{aligned}
$$

By means of Clément's projection $P_{C}$ and the fact that $a\left(\mathbf{j}_{h}, \mathbf{q}\right)=0$ for $\mathbf{q} \in$ $R T_{0}\left(\Omega ; \mathcal{T}_{k}\right) \cap H^{0}$ (div; $\Omega$ ), we obtain an upper bound for the solenoidal part of the flux error. Let $\mathbf{j}_{e}^{0}=\mathbf{c u r l} \psi$. Then, observing (1.6), (3.1b) and (3.1c), an upper bound for $\left\|\mathbf{j}_{e}^{0}\right\|_{\text {div }}$ results from

$$
\begin{aligned}
& \left\|\mathbf{j}_{e}^{0}\right\|_{\text {div }}^{2}=r\left(\mathbf{j}_{e}^{0}\right)=\sum_{e \in \mathcal{E}_{k}} \int_{e}\left(\left[a^{-1} \tilde{\mathbf{j}}_{h} \cdot \mathbf{t}_{e}\right]_{J}\right) \psi d \sigma \\
& =\sum_{e \in \mathcal{E}_{k}} \int_{e}\left(\left[a^{-1} \tilde{\mathbf{j}}_{h} \cdot \mathbf{t}_{e}\right]_{J}\right)\left(\psi-P_{C} \psi\right) d \sigma+\sum_{e \in \mathcal{E}_{k}} \int_{e}\left[a^{-1}\left(\tilde{\mathbf{j}}_{h}-\mathbf{j}_{h}\right) \cdot \mathbf{t}_{e}\right]_{J} P_{C} \psi d \sigma \\
& \leq \sum_{e \in \mathcal{E}_{k}}\left(\alpha_{e} h_{e} \int_{e}\left[a^{-1} \tilde{\mathbf{j}}_{h} \cdot \mathbf{t}_{e}\right]_{J}^{2} d \sigma\right)^{1 / 2}\left(\frac{1}{\alpha_{e} h_{e}} \int_{e}\left(\psi-P_{C} \psi\right)^{2} d \sigma\right)^{1 / 2} \\
& \quad+a\left(\mathbf{j}_{h}-\tilde{\mathbf{j}}_{h}, \operatorname{curl} P_{C} \psi\right) \\
& \leq\left\|\mathbf{j}_{e}^{0}\right\|_{\operatorname{div}}\left(\sqrt{\kappa_{K}} \hat{C}_{0 ; C}\left\|\left[a^{-1} \tilde{\mathbf{j}}_{h} \cdot \mathbf{t}_{e}\right]_{J}\right\|_{0 ; \mathcal{E}_{k}}+C_{1 ; C} \sqrt{\kappa_{D}}\left\|\tilde{\mathbf{j}}_{h}-\mathbf{j}_{h}\right\|_{\text {div }}\right) .
\end{aligned}
$$

On the other hand, taking into account (3.5a) and (3.5b), a lower bound for $\left\|\mathbf{j}_{e}^{0}\right\|_{\text {div }}$ can be established by means of the quadratic bubble functions $\Phi_{e}$ associated with the midpoints of the edges (cf. (3.3)):

$$
\begin{aligned}
C_{0 ; K}^{-2}\left\|\left[a^{-1} \tilde{\mathbf{j}}_{h} \cdot \mathbf{t}_{e}\right]_{J}\right\|_{0 ; e}^{2} & \leq \int_{e}\left[a^{-1} \tilde{\mathbf{j}}_{h} \cdot \mathbf{t}_{e}\right]_{J}^{2} \Phi_{e} d \sigma \\
& =\int_{e}\left[a^{-1} \tilde{\mathbf{j}}_{h} \cdot \mathbf{t}_{e}\right]_{J} \cdot\left(\left[a^{-1} \tilde{\mathbf{j}}_{h} \cdot \mathbf{t}_{e}\right]_{J} \Phi_{e}\right) d \sigma \\
& =-\int_{D_{e}} \mathbf{c u r l} \cdot\left(\left[a^{-1} \tilde{\mathbf{j}}_{h} \cdot \mathbf{t}_{e}\right]_{J}^{D_{e}} \Phi_{e}\right) \cdot\left(a^{-1}\left(\tilde{\mathbf{j}}_{h}-\mathbf{j}\right)\right) d x \\
& \leq\left\|a^{-1 / 2} \mathbf{j}_{e}^{0}\right\|_{0 ; D_{e}} \cdot\left\|\hat{a}^{-1 / 2} \nabla\left(\left[a^{-1} \tilde{\mathbf{j}}_{h} \cdot \mathbf{t}_{e}\right]_{J}^{D_{e}} \Phi_{e}\right)\right\|_{0 ; D_{e}} \\
& \leq\left(\alpha_{e} h_{e}\right)^{-1 / 2} C_{1 ; K}\left\|a^{-1 / 2} \mathbf{j}_{e}^{0}\right\|_{0 ; D_{e}} \cdot\left\|\left[a^{-1} \tilde{\mathbf{j}}_{h} \cdot \mathbf{t}_{e}\right]_{J}\right\|_{0 ; e}
\end{aligned}
$$

whence

$$
\left\|\left[a^{-1} \tilde{\mathbf{j}}_{h} \cdot \mathbf{t}_{e}\right]_{J}\right\|_{0 ; \mathcal{E}_{k}} \leq \sqrt{3} C_{0 ; K}^{2} C_{1 ; K}\left\|\mathbf{j}_{e}^{0}\right\|_{\text {div }} .
$$

We recall that the extension $\left[a^{-1} \tilde{\mathbf{J}}_{h} \cdot \mathbf{t}_{e}\right]_{J}^{D_{e}}$ is defined according to (3.6). The assertion is an immediate consequence of (3.10) and (3.11). 
In order to obtain a sharp estimate for the total flux error, in a second step we have to consider $\mathbf{j}_{e}^{1}$. It is easy to see that in the special case of a vanishing Helmholtz term

$$
\int_{\Omega} \operatorname{div}\left(\mathbf{j}-\tilde{\mathbf{j}}_{h}\right) v d x=\int_{\Omega}\left(f-\Pi_{0} f\right) v d x, \quad v \in L^{2}(\Omega)
$$

whence $\left\|\operatorname{div}\left(\mathbf{j}-\tilde{\mathbf{j}}_{h}\right)\right\|_{0}=\left\|f-\Pi_{0} f\right\|_{0}$. Because $\operatorname{div} \mathbf{j}_{e}=\operatorname{div} \mathbf{j}_{e}^{1}$, (3.9) readily provides a lower and an upper bound for $\left\|\mathbf{j}_{e}^{1}\right\|_{\text {div }}$.

However, in the general case $b \not \equiv 0$ the situation is more complicated, and the errors $\left\|u_{e}\right\|_{0}$ in the primal variable $u$ and in the divergence part of the flux error $\left\|\mathbf{j}_{e}^{1}\right\|_{\text {div }}$ are coupled.

Lemma 3.5. The following inequalities hold true:

$$
\begin{aligned}
\left\|\operatorname{div} \mathbf{j}_{e}^{1}\right\|_{0 ; T} & \leq\left\|f-\Pi_{0} f\right\|_{0 ; T}+b_{1}^{T}\left\|u_{e}\right\|_{0 ; T}, \\
\left\|f-\Pi_{0} f\right\|_{0 ; T} & \leq b_{1}^{T}\left\|u_{e}\right\|_{0 ; T}+\left\|\operatorname{div} \mathbf{j}_{e}^{1}\right\|_{0 ; T} .
\end{aligned}
$$

Proof. The second equation of the variational problem (2.1) states that

$$
\int_{\Omega} \operatorname{div} \mathbf{j}_{e} v d x+\int_{\Omega} b u_{e} v d x=\int_{\Omega}\left(f-\Pi_{0} f\right) v d x, \quad v \in L^{2}(\Omega),
$$

and we conclude by a straightforward application of the triangle inequality.

Finally, we will focus our attention on the error in the primal variable with respect to the $L^{2}$-norm $\left\|u-\tilde{u}_{h}\right\|_{0}$. For that purpose, we consider the $L^{2}$-projection of $u$ onto the space of piecewise constant functions and use the following result:

$$
\left\|\Pi_{0} u-u_{h}\right\|_{0}^{2} \leq C_{\text {sup }}^{2} \sum_{T \in \mathcal{T}_{k}} h_{T}^{2}\left\|a^{-1}\left(\mathbf{j}-\mathbf{j}_{h}\right)\right\|_{\mathrm{div} ; T}^{2},
$$

where the constant $C_{\text {sup }}$ only depends on the geometry of the initial triangulation and on the ratio of the local bounds of the coefficients in (1.1). In case of the Poisson equation, this result is well established (cf., e.g., [2, 12]). In the general case, it can be proved assuming a discrete $\mathrm{H}^{2}$-regularity and using some duality techniques (cf., e.g., [12, Remark 2.16]).

Lemma 3.6. There exist constants $c_{u_{e}}, c_{\mathbf{j}_{e}}, C_{u_{e}}, C_{\mathbf{j}_{e}}, C_{i t}^{1}>0$, independent of the refinement level, such that

$$
\begin{aligned}
c_{u_{e}}\left\|u_{e}\right\|_{0} \leq & \left\|a^{-1} \tilde{\boldsymbol{\jmath}}_{h}\right\|_{0 ; \mathcal{T}_{k}}+c_{\mathbf{j}_{e}}\left\|a^{-1} \mathbf{j}_{e}\right\|_{\text {div } ; \mathcal{T}_{k}} \\
& +C_{i t}^{1}\left(\left\|u_{h}-\tilde{u}_{h}\right\|_{0}+\left\|a^{-1}\left(\mathbf{j}_{h}-\tilde{\boldsymbol{\jmath}}_{h}\right)\right\|_{\mathrm{div} ; \mathcal{T}_{k}}\right), \\
\left\|a^{-1} \tilde{\boldsymbol{\jmath}}_{h}\right\|_{0 ; \mathcal{T}_{k} \leq} \leq & \left(C_{u_{e}}\left\|u_{e}\right\|_{0}+C_{\mathbf{j}_{e}}\left\|a^{-1} \mathbf{j}_{e}\right\|_{0 ; \mathcal{T}_{k}}\right),
\end{aligned}
$$

where the weighted norms are given by $\|\cdot\|_{0 ; \mathcal{T}_{k}}^{2}:=\sum_{T \in \mathcal{T}_{k}} h_{T}^{2}\|\cdot\|_{0 ; T}^{2}$ and $\|\cdot\|_{\text {div; }}^{2}:=$ $\sum_{T \in \mathcal{T}_{k}} h_{T}^{2}\|\cdot\|_{\mathrm{div} ; T}^{2} \cdot$ 
Proof. Since $\mathbf{j}=-a \nabla u$, in view of (3.3) and (3.12) we obtain

$$
\begin{aligned}
\left\|u-\tilde{u}_{h}\right\|_{0} \leq\left\|u-\Pi_{0} u\right\|_{0}+\left\|\Pi_{0} u-\tilde{u}_{h}\right\|_{0} \\
\leq C_{\text {proj }}\left(\sum_{T \in \mathcal{T}_{k}} h_{T}^{2}\|\nabla u\|_{0 ; T}^{2}\right)^{1 / 2}+C_{\text {sup }}\left\|a^{-1}\left(\mathbf{j}-\mathbf{j}_{h}\right)\right\|_{\operatorname{div} ; \mathcal{T}_{k}}+\left\|u_{h}-\tilde{u}_{h}\right\|_{0} \\
\leq \quad C_{\text {proj }}\left(\left\|a^{-1} \tilde{\mathbf{j}}_{h}\right\|_{0 ; \mathcal{T}_{k}}+\left\|a^{-1}\left(\mathbf{j}-\tilde{\mathbf{j}}_{h}\right)\right\|_{0 ; \mathcal{T}_{k}}\right) \\
\quad+C_{\text {sup }}\left(\left\|a^{-1}\left(\mathbf{j}-\tilde{\mathbf{j}}_{h}\right)\right\|_{\text {div } ; \mathcal{T}_{k}}+\left\|a^{-1}\left(\mathbf{j}_{h}-\tilde{\mathbf{j}}_{h}\right)\right\|_{\text {div } ; \mathcal{T}_{k}}\right)+\left\|u_{h}-\tilde{u}_{h}\right\|_{0} .
\end{aligned}
$$

The constants $c_{\mathbf{j}_{e}}, c_{u_{e}}$ and $C_{i t}^{1}>0$ are defined as $c_{\mathbf{j}_{e}}:=1+C_{\text {sup }} C_{\text {proj }}^{-1}, c_{u_{e}}:=C_{\text {proj }}^{-1}$ and $C_{i t}^{1}:=C_{\text {proj }}^{-1} \max \left(1, C_{\text {sup }}\right)$.

It remains to establish an upper bound for $\left\|a^{-1} \tilde{\mathbf{J}}_{h}\right\|_{0 ; \mathcal{T}_{k}}$. This can be achieved using the cubic bubble function $\phi_{T}$ (cf. (3.3)) and observing (3.4a), (3.4b):

$$
\begin{aligned}
C_{0 ; D}^{-2} & \int_{T}\left|a^{-1} \tilde{\mathbf{j}}_{h}\right|^{2} d x \leq \int_{T}\left(a^{-1} \tilde{\mathbf{j}}_{h}+\nabla u\right) \cdot\left(\phi_{T} a^{-1} \tilde{\mathbf{j}}_{h}\right) d x-\int_{T} \nabla u \cdot\left(\phi_{T} a^{-1} \tilde{\mathbf{j}}_{h}\right) d x \\
& =\int_{T} \operatorname{div}\left(\phi_{T} a^{-1} \tilde{\mathbf{j}}_{h}\right)\left(u-\tilde{u}_{h}\right) d x+\int_{T} a^{-1}\left(\tilde{\mathbf{J}}_{h}-\mathbf{j}\right) \cdot\left(\phi_{T} a^{-1} \tilde{\mathbf{j}}_{h}\right) d x \\
& \leq\left\|\tilde{u}_{h}-u\right\|_{0 ; T} \cdot\left\|\operatorname{div}\left(\phi_{T} a^{-1} \tilde{\mathbf{J}}_{h}\right)\right\|_{0 ; T}+\left\|a^{-1}\left(\tilde{\mathbf{j}}_{h}-\mathbf{j}\right)\right\|_{0 ; T} \cdot\left\|\phi_{T} a^{-1} \tilde{\mathbf{j}}_{h}\right\|_{0 ; T} \\
& \leq\left(h_{T}^{-1} C_{1 ; D}\left\|\tilde{u}_{h}-u\right\|_{0 ; T}+\left\|a^{-1}\left(\tilde{\mathbf{J}}_{h}-\mathbf{j}\right)\right\|_{0 ; T}\right)\left\|a^{-1} \tilde{\mathbf{j}}_{h}\right\|_{0 ; T} .
\end{aligned}
$$

This local inequality holds true for all elements $T \in \mathcal{T}_{k}$. Therefore, we obtain the global estimate

$$
\left\|a^{-1} \tilde{\mathbf{j}}_{h}\right\|_{0 ; \mathcal{T}_{k}} \leq C_{u_{e}}\left\|\tilde{u}_{h}-u\right\|_{0}+C_{\mathbf{j}_{e}}\left\|a^{-1}\left(\mathbf{j}-\tilde{\mathbf{j}}_{h}\right)\right\|_{0 ; \mathcal{T}_{k}},
$$

where $C_{u_{e}}:=\sqrt{2} C_{0 ; D}^{2} C_{1 ; D}$ and $C_{\mathbf{j}_{e}}:=\sqrt{2} C_{0 ; D}^{2}$.

If we take into account the definition of the residual based error estimator given by (2.2) and the results of Lemmas 3.4, 3.5, and 3.6, the proof of Theorem 2.1 can be readily given:

Proof of Theorem 2.1. By means of Lemmas 3.4, 3.5, and 3.6, we get

$$
\begin{aligned}
\| f- & \Pi_{0} f\left\|_{0}^{2}+\right\|\left[a^{-1} \tilde{\mathbf{j}}_{h} \mathbf{t}_{e}\right]_{J}\left\|_{0 ; \mathcal{E}_{k}}+\right\| a^{-1} \tilde{\mathbf{j}}_{h} \|_{0 ; \mathcal{T}_{k}} \\
\leq & 2\left(b_{1}^{2}+C_{u_{e}}^{2}\right)\left\|u_{e}\right\|_{0}^{2}+\left(c_{\mathbf{j}_{e}^{0}}^{-2}+2 h_{0}^{2} \alpha_{0}^{-1} C_{\mathbf{j}_{e}}^{2}\right)\left\|\mathbf{j}_{e}^{0}\right\|_{\text {div }}^{2} \\
& +2 \max \left(1, h_{0}^{2} \alpha_{0}^{-1} C_{\mathbf{j}_{e}}^{2}\right)\left\|\mathbf{j}_{e}^{1}\right\|_{\text {div }}^{2} .
\end{aligned}
$$

Hence, the first inequality of Theorem 2.1 holds true with

$$
c_{R}^{-1}:=\max \left(2,\left(c_{\mathbf{j}_{e}^{0}}^{-2}+2 h_{0}^{2} \alpha_{0}^{-1} C_{\mathbf{j}_{e}}^{2}\right), 2\left(b_{1}^{2}+C_{u_{e}}^{2}\right)\right) .
$$

To establish the upper bound for the error, we need an additional assumption on $h$. Assuming

$$
h_{0} \leq h_{\max }:=\left(\frac{3}{\alpha_{0}}\left(\frac{c_{\mathbf{j}_{e}}}{c_{u_{e}}}\right)^{2} \max \left(1, \alpha_{0}^{-1}\right)\left(1+4 C_{d i v}^{2} b_{1}^{2} \max \left(1, \alpha_{0}^{-1}\right)\right)\right)^{-1 / 2},
$$


by means of Lemmas 3.4, 3.5, and 3.6 we obtain

$$
\begin{aligned}
&\left\|u_{e}\right\|_{0}^{2}+\left\|\mathbf{j}_{e}\right\|_{\text {div }}^{2}=\left\|u_{e}\right\|_{0}^{2}+\left\|\mathbf{j}_{e}^{0}\right\|_{\text {div }}^{2}+\left\|\mathbf{j}_{e}^{1}\right\|_{\text {div }}^{2} \\
& \leq 4 C_{\mathbf{j}_{e}^{0}}^{2}\left\|\left[a^{-1} \tilde{\mathbf{j}}_{h} \cdot \mathbf{t}_{e}\right]_{J}\right\|_{0 ; \mathcal{E}_{k}}^{2}+4 C_{d i v}^{2} \max \left(1, \alpha_{0}^{-1}\right)\left\|f-\Pi_{0} f\right\|_{0}^{2} \\
&+3 c_{u_{e}}^{-2}\left(1+4 b_{1}^{2} C_{d i v}^{2} \max \left(1, \alpha_{0}^{-1}\right)\right)\left\|a^{-1} \tilde{\mathbf{j}}_{h}\right\|_{0 ; \mathcal{T}_{k}}^{2}+4\left(C_{i t}^{0}\right)^{2}\left\|\mathbf{j}_{h}-\tilde{\mathbf{j}}_{h}\right\|_{\text {div }}^{2} \\
&+3\left(c_{u_{e}}^{-1} C_{i t}^{1}\right)^{2}\left(1+4 b_{1}^{2} C_{d i v}^{2} \max \left(1, \alpha_{0}^{-1}\right)\right)\left(\left\|u_{h}-\tilde{u}_{h}\right\|_{0}+\left\|\mathbf{j}_{h}-\tilde{\mathbf{j}}_{h}\right\|_{\text {div } ; \mathcal{T}_{k}}\right)^{2} .
\end{aligned}
$$

Then, the upper bound holds true with

$$
C_{R}:=\max \left(3 c_{u_{e}}^{-2}\left(1+4 b_{1}^{2} C_{d i v}^{2} \max \left(1, \alpha_{0}^{-1}\right)\right), 4 C_{\mathbf{j}_{e}^{\mathbf{0}}}^{2}, 4 C_{d i v}^{2} \max \left(1, \alpha_{0}^{-1}\right)\right)
$$

and

$$
\begin{aligned}
& C_{i t}:=2 \max \left(3\left(\frac{C_{i t}^{1}}{c_{u_{e}}}\right)^{2}\right.\left(1+4 b_{1}^{2} C_{d i v}^{2} \max \left(1, \alpha_{0}^{-1}\right)\right) \\
&\left.2\left(C_{i t}^{0}\right)^{2}+\left(\frac{C_{i t}^{1}}{c_{\mathbf{j}_{e}}} \frac{\max \left(1, \alpha_{1}\right)}{\max \left(1, \alpha_{0}^{-1}\right)}\right)^{2}\right) .
\end{aligned}
$$

Remark. Note that we even get an upper bound if

$$
h_{0}<C_{\infty}:=\left(\sqrt{\frac{6}{\alpha_{0}}} b_{1} C_{d i v} \frac{c_{\mathbf{j}_{e}}}{c_{u_{e}}} \max \left(1, \alpha_{0}^{-1}\right)\right)^{-1} .
$$

In case $b \equiv 0$, we define $C_{\infty}:=\infty$, and thus we have no limitation on $h_{0}$. If $h_{0}$ tends to $C_{\infty}$, the upper bounds in the theorem tend to $\infty$ as well.

\section{THE HIERARCHICAL BASIS ERROR ESTIMATOR}

As indicated in Section 2, the idea behind the hierarchical type a posteriori error estimator consists in an approximation of the error equation with respect to the higher order mixed ansatz spaces $R T_{1}\left(\Omega ; \mathcal{T}_{k}\right)$ and $W_{1}\left(\Omega ; \mathcal{T}_{k}\right)$, followed by an appropriate localization in terms of a hierarchical two-level splitting. In particular, introducing the local spaces

$$
\widehat{R T}_{1}^{0}(T):=\left\{\mathbf{p} \in R T_{1}(T)|\quad \mathbf{p}=\mathbf{q}|_{T}, \mathbf{q} \in \widehat{R T}_{1}^{0}\left(\Omega ; \mathcal{T}_{k}\right)\right\}, \quad T \in \mathcal{T}_{k}, k \geq 0,
$$

and $\widehat{R T}_{1}(T):=\widehat{R T}_{1}^{0}(T) \oplus \widehat{R T}_{1}^{1}(T)$, where $\widehat{R T}_{1}^{1}(T)$ is given as in Section 2, the following strengthened Cauchy-Schwarz inequalities (4.1) will play an important role for the derivation of the error estimator:

$$
\begin{array}{ll}
\left.a\right|_{T}\left(\mathbf{q}^{0}, \mathbf{q}^{1}\right)^{2} \leq\left.\left.\eta_{0}^{2} a\right|_{T}\left(\mathbf{q}^{0}, \mathbf{q}^{0}\right) a\right|_{T}\left(\mathbf{q}^{1}, \mathbf{q}^{1}\right), & \mathbf{q}^{0} \in R T_{0}(T), \mathbf{q}^{1} \in \widehat{R T}_{1}(T), \\
\left.a\right|_{T}\left(\hat{\mathbf{q}}^{0}, \hat{\mathbf{q}}^{1}\right)^{2} \leq\left.\left.\eta_{1}^{2} a\right|_{T}\left(\hat{\mathbf{q}}^{0}, \hat{\mathbf{q}}^{0}\right) a\right|_{T}\left(\hat{\mathbf{q}}^{1}, \hat{\mathbf{q}}^{1}\right), & \hat{\mathbf{q}}^{\nu} \in \widehat{R T}_{1}^{\nu}(T), 0 \leq \nu \leq 1,
\end{array}
$$

with $\eta_{\nu}^{2}<1,0 \leq \nu \leq 1$, being independent of the refinement level. The inequalities (4.1) are an easy consequence of the affine equivalence of the Raviart-Thomas elements and the shape regularity of the triangulations.

The system (2.3) cannot be solved locally, and therefore the approximation $\left(e_{\mathbf{j}}, e_{u}\right)$ is not suited for an easily computable error estimator. Consequently, the main idea of our proposed error estimator consists in the replacement of the original 
bilinear form $a(\cdot, \cdot)$ by a modified bilinear form $\tilde{a}(\cdot, \cdot)$. According to the splitting of $R T_{1}\left(\Omega ; \mathcal{T}_{k}\right)$ the vector fields $\mathbf{q}, \mathbf{p} \in R T_{1}\left(\Omega ; \mathcal{T}_{k}\right)$ are decomposed as follows:

$$
\mathbf{q}=\mathbf{q}^{0}+\hat{\mathbf{q}}^{0}+\hat{\mathbf{q}}^{1}, \quad \mathbf{p}=\mathbf{p}^{0}+\hat{\mathbf{p}}^{0}+\hat{\mathbf{p}}^{1}
$$

where $\mathbf{q}^{0}, \mathbf{p}^{0} \in R T_{0}\left(\Omega ; \mathcal{T}_{k}\right), \hat{\mathbf{q}}^{0}, \hat{\mathbf{p}}^{0} \in \widehat{R T}_{1}^{0}\left(\Omega ; \mathcal{T}_{k}\right)$ and $\hat{\mathbf{q}}^{1}, \hat{\mathbf{p}}^{1} \in \widehat{R T}_{1}^{1}\left(\Omega ; \mathcal{T}_{k}\right)$. Then, the modified bilinear form $\tilde{a}(\mathbf{q}, \mathbf{p})$ is defined as follows:

$$
\tilde{a}(\mathbf{q}, \mathbf{p}):=a\left(\mathbf{q}^{0}, \mathbf{p}^{0}\right)+a\left(\hat{\mathbf{q}}^{0}, \hat{\mathbf{p}}^{0}\right)+a\left(\hat{\mathbf{q}}^{1}, \hat{\mathbf{p}}^{1}\right) .
$$

It is easy to see that $\tilde{a}(\cdot, \cdot)$ is orthogonal with respect to the decomposition of the ansatz space of the flux. A simple consequence of the strengthened CauchySchwarz inequalities (4.1) and Young's inequality is the equivalence of the bilinear forms $a(\cdot, \cdot)$ and $\tilde{a}(\cdot, \cdot)$ :

$$
\left.c_{R T}^{2} \tilde{a}\right|_{T}(\mathbf{q}, \mathbf{q}) \leq\left. a\right|_{T}(\mathbf{q}, \mathbf{q}) \leq\left. C_{R T}^{2} \tilde{a}\right|_{T}(\mathbf{q}, \mathbf{q}), \quad \mathbf{q} \in R T_{1}(T),
$$

with constants $0<c_{R T} \leq C_{R T}$ independent of $T \in \mathcal{T}_{k}, k \geq 1$. A detailed proof of (4.2) can be found in [32, Lemma 4.6]. Now, we consider a modified discrete variational problem which is obtained from $(2.3)$ if we replace the bilinear form $a(\cdot, \cdot)$ by $\tilde{a}(\cdot, \cdot)$ : Find $\left(\tilde{e}_{\mathbf{j}}, \tilde{e}_{u}\right) \in R T_{1}\left(\Omega ; \mathcal{T}_{k}\right) \times W_{1}\left(\Omega ; \mathcal{T}_{k}\right)$ such that

$$
\begin{aligned}
\tilde{a}\left(\tilde{e}_{\mathbf{j}}, \mathbf{q}\right)+b\left(\mathbf{q}, \tilde{e}_{u}\right) & =r(\mathbf{q}), & & \mathbf{q} \in R T_{1}\left(\Omega ; \mathcal{T}_{k}\right), \\
b\left(\tilde{e}_{\mathbf{j}}, v\right)-c\left(\tilde{e}_{u}, v\right) & =-\left(f-\Pi_{0} f, v\right)_{0}, & & v \in W_{1}\left(\Omega ; \mathcal{T}_{k}\right) .
\end{aligned}
$$

The following theorem states the equivalence of the solutions of the saddle point problems (2.3) and (4.3). By means of (2.4) and Theorem 2.2, $\left\|\tilde{e}_{\mathbf{j}}\right\|_{\text {div }}$ provides sharp upper and lower bounds for the error in the flux.

Theorem 4.1. The solutions $\left(e_{\mathbf{j}}, e_{u}\right)$ and $\left(\tilde{e}_{\mathbf{j}}, \tilde{e}_{u}\right) \in R T_{1}\left(\Omega ; \mathcal{T}_{k}\right) \times W_{1}\left(\Omega ; \mathcal{T}_{k}\right)$ of the discrete variational problems (2.3) and (4.3) are equivalent in the sense that there exist constants $0<c_{j ; R T} \leq C_{j ; R T}$ and $c_{d ; R T}, c_{u ; R T}, C_{d ; R T}, C_{u ; R T}>0$, independent of $\mathcal{T}_{k}, k \geq 1$, such that

$$
\begin{gathered}
c_{j ; R T}^{2} \tilde{a}\left(\tilde{e}_{\mathbf{j}}, \tilde{e}_{\mathbf{j}}\right) \leq a\left(e_{\mathbf{j}}, e_{\mathbf{j}}\right) \leq C_{j ; R T}^{2} \tilde{a}\left(\tilde{e}_{\mathbf{j}}, \tilde{e}_{\mathbf{j}}\right) \\
\left\|\operatorname{div} \tilde{e}_{\mathbf{j}}\right\|_{0} \leq\left\|\operatorname{div} e_{\mathbf{j}}\right\|_{0}+c_{d ; R T} a\left(e_{\mathbf{j}}, e_{\mathbf{j}}\right)^{1 / 2} \\
\left\|\operatorname{div} e_{\mathbf{j}}\right\|_{0} \leq\left\|\operatorname{div} \tilde{e}_{\mathbf{j}}\right\|_{0}+C_{d ; R T} \tilde{a}\left(\tilde{e}_{\mathbf{j}}, \tilde{e}_{\mathbf{j}}\right)^{1 / 2} \\
\left\|\tilde{e}_{u}\right\|_{0} \leq\left\|e_{u}\right\|_{0}+c_{u ; R T} a\left(e_{\mathbf{j}}, e_{\mathbf{j}}\right)^{1 / 2} \\
\left\|e_{u}\right\|_{0} \leq\left\|\tilde{e}_{u}\right\|_{0}+C_{u ; R T} \tilde{a}\left(\tilde{e}_{\mathbf{j}}, \tilde{e}_{\mathbf{j}}\right)^{1 / 2}
\end{gathered}
$$

Proof. In view of (2.3) and (4.3) we compare the solutions $e_{\mathbf{j}}$ and $\tilde{e}_{\mathbf{j}}$ and obtain

$$
a\left(e_{\mathbf{j}}-\tilde{e}_{\mathbf{j}}, e_{\mathbf{j}}-\tilde{e}_{\mathbf{j}}\right)+c\left(e_{u}-\tilde{e}_{u}, e_{u}-\tilde{e}_{u}\right)=\tilde{a}\left(\tilde{e}_{\mathbf{j}}, e_{\mathbf{j}}-\tilde{e}_{\mathbf{j}}\right)-a\left(\tilde{e}_{\mathbf{j}}, e_{\mathbf{j}}-\tilde{e}_{\mathbf{j}}\right)
$$

as well as

$$
\tilde{a}\left(e_{\mathbf{j}}-\tilde{e}_{\mathbf{j}}, e_{\mathbf{j}}-\tilde{e}_{\mathbf{j}}\right)+c\left(e_{u}-\tilde{e}_{u}, e_{u}-\tilde{e}_{u}\right)=\tilde{a}\left(e_{\mathbf{j}}, e_{\mathbf{j}}-\tilde{e}_{\mathbf{j}}\right)-a\left(e_{\mathbf{j}}, e_{\mathbf{j}}-\tilde{e}_{\mathbf{j}}\right) .
$$

Observing (4.2) and the fact that $c(\cdot, \cdot)$ is positive semidefinite, by straightforward calculations we get

$$
\begin{aligned}
& a\left(e_{\mathbf{j}}-\tilde{e}_{\mathbf{j}}, e_{\mathbf{j}}-\tilde{e}_{\mathbf{j}}\right)^{1 / 2} \leq\left(C_{R T}+\frac{1}{c_{R T}}\right) \tilde{a}\left(\tilde{e}_{\mathbf{j}}, \tilde{e}_{\mathbf{j}}\right)^{1 / 2} \\
& \tilde{a}\left(e_{\mathbf{j}}-\tilde{e}_{\mathbf{j}}, e_{\mathbf{j}}-\tilde{e}_{\mathbf{j}}\right)^{1 / 2} \leq\left(C_{R T}+\frac{1}{c_{R T}}\right) a\left(e_{\mathbf{j}}, e_{\mathbf{j}}\right)^{1 / 2}
\end{aligned}
$$


The triangle inequality applied to $a\left(e_{\mathbf{j}}, e_{\mathbf{j}}\right)^{1 / 2}$ and $\tilde{a}\left(\tilde{e}_{\mathbf{j}}, \tilde{e}_{\mathbf{j}}\right)^{1 / 2}$ proves $(4.4 \mathrm{a})$ with $C_{j ; R T}:=2 C_{R T}+c_{R T}^{-1}$ and $c_{j ; R T}:=\left(C_{R T}+2 c_{R T}^{-1}\right)^{-1}$.

Recalling that $\operatorname{div} R T_{1}\left(\Omega ; \mathcal{T}_{k}\right)=W_{1}\left(\Omega ; \mathcal{T}_{k}\right)$ and

$$
b\left(\tilde{e}_{\mathbf{j}}-e_{\mathbf{j}}, v\right)=c\left(\tilde{e}_{u}-e_{u}, v\right), \quad v \in W_{1}\left(\Omega ; \mathcal{T}_{k}\right),
$$

we see that $(4.4 \mathrm{~b})$ follows from

$$
\begin{aligned}
\left\|\operatorname{div}\left(\tilde{e}_{\mathbf{j}}-e_{\mathbf{j}}\right)\right\|_{0} & =\sup _{\substack{v \in W_{1}\left(\Omega ; \mathcal{T}_{k}\right) \\
v \neq 0}} \frac{b\left(\tilde{e}_{\mathbf{j}}-e_{\mathbf{j}}, v\right)}{\|v\|_{\mathbf{j}}, \Omega} \leq \sqrt{\beta_{1}} c\left(e_{u}-\tilde{e}_{u}, e_{u}-\tilde{e}_{u}\right)^{1 / 2} \\
& =\sqrt{\beta_{1}}\left(\tilde{a}\left(e_{\mathbf{j}}-\tilde{e}_{\mathbf{j}}, \tilde{e}_{\mathbf{j}}\right)-a\left(e_{\mathbf{j}}-\tilde{e}_{\mathbf{j}}, e_{\mathbf{j}}\right)\right)^{1 / 2} \\
& \leq \sqrt{\beta_{1}} \sqrt{2\left(C_{R T}+c_{R T}^{-1}\right)} \tilde{a}\left(\tilde{e}_{\mathbf{j}}, \tilde{e}_{\mathbf{j}}\right)^{1 / 4} a\left(e_{\mathbf{j}}, e_{\mathbf{j}}\right)^{1 / 4}
\end{aligned}
$$

By means of the triangle inequality and (4.4a) we conclude with that $C_{d ; R T}^{2}:=$ $2 \beta_{1} C_{j ; R T}\left(C_{R T}+c_{R T}^{-1}\right)$ and $c_{d ; R T}^{2}:=2 c_{j ; R T}^{-1} \beta_{1}\left(C_{R T}+c_{R T}^{-1}\right)$.

For the proof of $(4.4 \mathrm{c})$ we note that for all $\mathbf{q} \in R T_{1}\left(\Omega ; \mathcal{T}_{k}\right)$ we have

$$
b\left(\mathbf{q}, e_{u}-\tilde{e}_{u}\right)=\tilde{a}\left(\tilde{e}_{\mathbf{j}}, \mathbf{q}\right)-a\left(e_{\mathbf{j}}, \mathbf{q}\right) .
$$

Taking into account the inequality

$$
\|v\|_{0} \leq \beta_{\text {div }} \sup _{\substack{\mathbf{q} \in R T_{1}\left(\Omega ; \mathcal{T}_{k}\right) \\ \mathbf{q} \neq 0}} \frac{b(\mathbf{q}, v)}{\|\mathbf{q}\|_{\text {div }}}, \quad v \in W_{1}\left(\Omega ; \mathcal{T}_{k}\right),
$$

and (4.2), (4.4a) and (4.5), we obtain

$$
\begin{aligned}
&\left\|e_{u}-\tilde{e}_{u}\right\|_{0} \leq \alpha_{0}^{-1 / 2} \beta_{\text {div }}\left(1+c_{R T}^{-1} c_{j ; R T}^{-1}\right) a\left(e_{\mathbf{j}}, e_{\mathbf{j}}\right)^{1 / 2}, \\
&\left\|e_{u}-\tilde{e}_{u}\right\|_{0} \leq \alpha_{0}^{-1 / 2} \beta_{\text {div }}\left(C_{j ; R T}+c_{R T}^{-1}\right) \tilde{a}\left(\tilde{e}_{\mathbf{j}}, \tilde{e}_{\mathbf{j}}\right)^{1 / 2} .
\end{aligned}
$$

A simple consequence of Theorem 4.1 is the existence of constants $0<c_{a^{-1}} \leq$ $C_{a^{-1}}$ such that

$$
c_{a^{-1}}^{2}\left(\tilde{a}\left(\tilde{e}_{\mathbf{j}}, \tilde{e}_{\mathbf{j}}\right)+\left\|\operatorname{div} \tilde{e}_{\mathbf{j}}\right\|_{0 ; \Omega}^{2}\right) \leq\left\|e_{j}\right\|_{\operatorname{div}}^{2} \leq C_{a^{-1}}^{2}\left(\tilde{a}\left(\tilde{e}_{\mathbf{j}}, \tilde{e}_{\mathbf{j}}\right)+\left\|\operatorname{div} \tilde{e}_{\mathbf{j}}\right\|_{0 ; \Omega}^{2}\right),
$$

where $c_{a^{-1}}^{-2}:=\max \left(2,2 c_{d ; R T}^{2}+c_{j ; R T}^{-2}\right)$ and $C_{a^{-1}}^{2}:=\max \left(2,2 C_{d ; R T}^{2}+C_{j ; R T}^{2}\right)$.

To prove the upper and lower bounds for the error estimator defined by (2.10), we have to show the equivalence between $\tilde{a}\left(\tilde{e}_{\mathbf{j}}, \tilde{e}_{\mathbf{j}}\right)+\left\|\operatorname{div} \tilde{e}_{\mathbf{j}}\right\|_{0 ; \Omega}^{2}$ and $\eta_{H}^{2}$. A first step in this direction is to consider the saddle point problem (4.3) in more detail. According to the hierarchical splitting of the spaces $R T_{1}\left(\Omega ; \mathcal{T}_{k}\right)$ and $W_{1}\left(\Omega ; \mathcal{T}_{k}\right)$, (4.3) can be rewritten in terms of three independent subproblems. We decompose $\tilde{e}_{\mathbf{j}}$ and $\tilde{e}_{u}$ as follows:

$$
\begin{aligned}
& \tilde{e}_{\mathbf{j}}=\tilde{e}_{\mathbf{j}_{0}}+\tilde{e}_{\mathbf{j}_{1}}^{0}+\tilde{e}_{\mathbf{j}_{1}}^{1}, \quad \tilde{e}_{\mathbf{j}_{0}} \in R T_{0}\left(\Omega ; \mathcal{T}_{k}\right), \tilde{e}_{\mathbf{j}_{1}}^{0} \in \widehat{R T}_{1}^{0}\left(\Omega ; \mathcal{T}_{k}\right), \tilde{e}_{\mathbf{j}_{1}}^{1} \in \widehat{R T}_{1}^{1}\left(\Omega ; \mathcal{T}_{k}\right), \\
& \tilde{e}_{u}=\tilde{e}_{u_{0}}+\tilde{e}_{u_{1}}, \quad \tilde{e}_{u_{0}} \in W_{0}\left(\Omega ; \mathcal{T}_{k}\right), \tilde{e}_{u_{1}} \in \widehat{W}_{1}\left(\Omega ; \mathcal{T}_{k}\right),
\end{aligned}
$$

and obtain three variational problems:

$$
\begin{gathered}
a\left(\tilde{e}_{\mathbf{j}_{0}}, \mathbf{q}\right)+b\left(\mathbf{q}, \tilde{e}_{u_{0}}\right)=r(\mathbf{q}), \quad \mathbf{q} \in R T_{0}\left(\Omega ; \mathcal{T}_{k}\right), \\
b\left(\tilde{e}_{\mathbf{j}_{0}}, v\right)-c\left(\tilde{e}_{u_{0}}, v\right)=0, \quad v \in W_{0}\left(\Omega ; \mathcal{T}_{k}\right), \\
a\left(\tilde{e}_{\mathbf{j}_{1}}^{0}, \mathbf{q}\right)=r(\mathbf{q}), \quad \mathbf{q} \in \widehat{R T}_{1}^{0}\left(\Omega ; \mathcal{T}_{k}\right),
\end{gathered}
$$




$$
\begin{aligned}
a\left(\tilde{e}_{\mathbf{j}_{1}}^{1}, \mathbf{q}\right)+b\left(\mathbf{q}, \tilde{e}_{u_{1}}\right) & =r(\mathbf{q}), & & \mathbf{q} \in \widehat{R T}_{1}^{1}\left(\Omega ; \mathcal{T}_{k}\right), \\
b\left(\tilde{e}_{\mathbf{j}_{1}}^{1}, v\right)-c\left(\tilde{e}_{u_{1}}, v\right) & =-(f, v)_{0}, & & v \in \widehat{W}_{1}\left(\Omega ; \mathcal{T}_{k}\right) .
\end{aligned}
$$

Again $r(\mathbf{q})$ denotes the residual. The solution $\left(\tilde{e}_{\mathbf{j}_{0}}, \tilde{e}_{u_{0}}\right)$ of (4.7) is zero only in case of a vanishing iteration error $\left(\tilde{\mathbf{j}}_{h}-\mathbf{j} h, \tilde{u}_{h}-u_{h}\right)$. An upper bound for $a\left(\tilde{e}_{\mathbf{j}_{0}}, \tilde{e}_{\mathbf{j}_{0}}\right)$ and $\left\|\operatorname{div} \tilde{e}_{\mathbf{j}_{0}}\right\|_{0 ; \Omega}^{2}$ can be easily established by means of the iteration error.

The variational problems (4.7) and (4.9) are indefinite saddle point problems. On the other hand, (4.8) represents a symmetric and positive definite system which can be decoupled by well known standard techniques (cf. [14]). The bilinear form $a(\cdot, \cdot)$ applied to the discrete space $\widehat{R T}_{1}^{0}\left(\Omega ; \mathcal{T}_{k}\right) \times \widehat{R T}_{1}^{0}\left(\Omega ; \mathcal{T}_{k}\right)$ can be seen as a bilinear form $\hat{a}(\cdot, \cdot)$ on $\hat{S}_{2}\left(\Omega ; \mathcal{T}_{k}\right) \times \hat{S}_{2}\left(\Omega ; \mathcal{T}_{k}\right)$. Let $\mathbf{q}, \mathbf{p} \in \widehat{R T}_{1}^{0}\left(\Omega ; \mathcal{T}_{k}\right)$ and $\phi, \psi \in \hat{S}_{2}\left(\Omega ; \mathcal{T}_{k}\right)$ with $\mathbf{q}=\operatorname{curl} \phi$ and $\mathbf{p}=\operatorname{curl} \psi$; then

$$
a(\mathbf{q}, \mathbf{p})=\sum_{T \in \mathcal{T}_{k}} \int_{T} a^{-1} \operatorname{curl} \phi \mathbf{c u r l} \psi d x=\sum_{T \in \mathcal{T}_{k}} \int_{T} \hat{a} \nabla \phi \nabla \psi d x=: \hat{a}(\phi, \psi) .
$$

The matrix $\hat{a}$ is defined by $\hat{a}_{11}:=\left(a^{-1}\right)_{22}, \hat{a}_{22}:=\left(a^{-1}\right)_{11}$ and $\hat{a}_{12}=\hat{a}_{21}:=$ $-\left(a^{-1}\right)_{12}$, and has the same eigenvalues as $a^{-1}$. Each element $\phi \in \hat{S}_{2}\left(\Omega ; \mathcal{T}_{k}\right)$ can be written as the direct sum of quadratic bubble functions associated with the edges of the triangulations according to $\phi=\sum_{e \in \mathcal{E}_{k}} \phi_{e}$, where $\phi_{e}, e \in \mathcal{E}_{k}$, is a multiple of the nodal basis function $\Phi_{e}$. The inequality

$$
\left.c_{\text {curl }} \sum_{i=1}^{3} \hat{a}\right|_{T}\left(\phi_{e_{i}}, \phi_{e_{i}}\right) \leq\left.\hat{a}\right|_{T}(\phi, \phi) \leq\left. C_{\text {curl }} \sum_{i=1}^{3} \hat{a}\right|_{T}\left(\phi_{e_{i}}, \phi_{e_{i}}\right), \quad T \in \mathcal{T}_{k},
$$

with constants $0<c_{\text {curl }} \leq C_{\text {curl }}$ states that the global problem (4.8) can be replaced by local ones. The coupling between different bubble functions is neglected and a single equation has to be solved for each edge (cf. (2.8) of Section 2):

$$
\hat{a}\left(\varphi_{e}, \Phi_{e}\right)=r\left(\operatorname{curl} \Phi_{e}\right) .
$$

Then, equivalence of $\sum_{e \in \mathcal{E}_{k}} \hat{a}\left(\varphi_{e}, \varphi_{e}\right)$ and $a\left(\tilde{e}_{\mathbf{j}_{1}}^{0}, \tilde{e}_{\mathbf{j}_{1}}^{0}\right)$ is guaranteed by (4.11).

It remains to examine (4.9). Due to the special structure of $\widehat{R T}_{1}^{1}\left(\Omega ; \mathcal{T}_{k}\right)$ and $\widehat{W}_{1}\left(\Omega ; \mathcal{T}_{k}\right)$, the global problem consists of independent local subproblems associated with the elements of the triangulation. For each element we have to solve the $4 \times 4$ saddle point problem (2.9), which can be further reduced to two $2 \times 2$ problems using an $L^{2}$-orthogonal basis of $\widehat{W}_{1}(T)$.

For the proof of Theorem 2.2 it remains to be shown that the flux of the solution of subproblem (4.7) is bounded by the iteration error independently of the refinement level. For this purpose we reconsider the residual:

$$
\begin{aligned}
r\left(\tilde{e}_{\mathbf{j}_{0}}\right) & =a\left(\tilde{e}_{\mathbf{j}_{0}}, \tilde{e}_{\mathbf{j}_{0}}\right)+c\left(\tilde{e}_{u_{0}}, \tilde{e}_{u_{0}}\right)=-a\left(\tilde{\mathbf{j}}_{h}, \tilde{e}_{\mathbf{j}_{0}}\right)-b\left(\tilde{e}_{\mathbf{j}_{0}}, \tilde{u}_{h}\right) \\
& =a\left(\mathbf{j}_{h}-\tilde{\mathbf{j}}_{h}, \tilde{e}_{\mathbf{j}_{0}}\right)+b\left(\tilde{e}_{\mathbf{j}_{0}}, u_{h}-\tilde{u}_{h}\right)=a\left(\mathbf{j}_{h}-\tilde{\mathbf{j}}_{h}, \tilde{e}_{\mathbf{j}_{0}}\right)+c\left(\tilde{e}_{u_{0}}, u_{h}-\tilde{u}_{h}\right) .
\end{aligned}
$$

In view of the equalities

$c\left(u_{h}-\tilde{u}_{h}, u_{h}-\tilde{u}_{h}\right)=b\left(\mathbf{j}_{h}-\tilde{\mathbf{j}}_{h}, u_{h}-\tilde{u}_{h}\right)$ and $\left\|\operatorname{div}\left(\tilde{e}_{\mathbf{j}_{0}}\right)\right\|_{0 ; \Omega}^{2}=b\left(\tilde{e}_{\mathbf{j}_{0}}, \operatorname{div}\left(\tilde{e}_{\mathbf{j}_{0}}\right)\right)$, we obtain

$$
\begin{aligned}
a\left(\tilde{e}_{\mathbf{j}_{0}}, \tilde{e}_{\mathbf{j}_{0}}\right)+c\left(\tilde{e}_{u_{0}}, \tilde{e}_{u_{0}}\right) & \leq\left\|\mathbf{j}_{h}-\tilde{\mathbf{j}}_{h}\right\|_{\text {div }}^{2} \\
\left\|\operatorname{div}\left(\tilde{e}_{\mathbf{j}_{0}}\right)\right\|_{0 ; \Omega}^{2} & \leq \beta_{1}\left\|\mathbf{j}_{h}-\tilde{\mathbf{j}}_{h}\right\|_{\text {div }}^{2}
\end{aligned}
$$


Proof of Theorem 2.2. Because of (2.4), (4.6) and (4.11), the assertion (2.11) is a direct consequence of the definition of the error estimator with constants $C_{\text {hier }}:=$ $C_{a^{-1}} \max \left(1, c_{\text {curl }}^{-1 / 2}\right)$ and $\Gamma_{\text {hier }}:=\beta_{\infty} \max \left(1, \sqrt{\beta_{1}}\right)+C_{a^{-1}} \sqrt{1+\beta_{1}}$ for the upper bounds of the error. The constants for the lower bounds are given by $c_{\text {hier }}:=$ $c_{a^{-1}} \min \left(1, C_{\text {cur1 }}^{-1 / 2}\right)$ and $\gamma_{\text {hier }}:=\beta_{\infty} \max \left(1, \sqrt{\beta_{1}}\right)$.

Remark. If we are interested in an error estimator for the error in the flux and in the primal variable, we have to take into account an additional term in the definition of the error estimator $\hat{\eta}_{H}$ :

$$
\begin{aligned}
\hat{\eta}_{H}^{2} & :=\sum_{T \in \mathcal{T}_{k}} \hat{\eta}_{H ; T}^{2}, \\
\hat{\eta}_{H ; T}^{2} & :=\tilde{\eta}_{H ; T}^{2}+\left\|\tilde{e}_{u_{1}}\right\|_{0 ; T}^{2}, \quad T \in \mathcal{T}_{k} .
\end{aligned}
$$

It is easy to see that the saturation assumption (S1 b) and (4.4c) as well as (2.11) guarantee that the error estimator $\hat{\eta}_{H}$ provides sharp lower and upper bounds for the total error $\left(\left\|u-\tilde{u}_{h}\right\|_{0}^{2}+\left\|\mathbf{j}-\tilde{\mathbf{j}}_{h}\right\|_{\text {div }}^{2}\right)^{1 / 2}$, if the iteration error is small enough.

\section{AN ERROR ESTIMATOR BASED ON LOCAL SUBPROBLEMS}

This section is devoted to the proof of Theorems 2.3 and 2.4.

Proof of Theorem 2.3. As a first step we consider $\hat{e}_{\mathbf{j}}-e_{\mathbf{j}}$. We recall that $\left(e_{\mathbf{j}}, e_{u}\right)$ denotes the discrete solution of (2.3). In general, the solution $\hat{e}_{\mathbf{j}} \in R T_{1}^{-1}\left(\Omega ; \mathcal{T}_{k}\right)$ is not contained in $H(\operatorname{div} ; \Omega)$. In the following, the bilinear forms $a(\cdot, \cdot)$ and $b(\cdot, \cdot)$ are extended to the nonconforming ansatz spaces in a natural way, i.e., the integrals over $\Omega$ are replaced by the sum of the integrals over the elements $T$ of the triangulation $\mathcal{T}_{k}$ :

$$
\begin{aligned}
& a\left(\hat{e}_{\mathbf{j}}-e_{\mathbf{j}}, \hat{e}_{\mathbf{j}}\right)=-b\left(\hat{e}_{\mathbf{j}}, \hat{e}_{u}-e_{u}\right)-\sum_{e \in \mathcal{E}_{k}} \int_{e}\left(\left[u_{D}\right]_{A}-\lambda_{R T_{1}}\right)\left[\mathbf{n}_{e} \cdot \hat{e}_{\mathbf{j}}\right]_{J} d \sigma \\
& =-c\left(\hat{e}_{u}, \hat{e}_{u}-e_{u}\right)+\left(f-\Pi_{0} f, \hat{e}_{u}-e_{u}\right)_{0} \\
& -\sum_{e \in \mathcal{E}_{k}} \int_{e}\left(\left[u_{D}\right]_{A}-\lambda_{R T_{1}}\right)\left[\mathbf{n}_{e} \cdot\left(\hat{e}_{\mathbf{j}}-e_{\mathbf{j}}\right)\right]_{J} d \sigma \\
& =-c\left(\hat{e}_{u}-e_{u}, \hat{e}_{u}-e_{u}\right)-b\left(e_{\mathbf{j}}, \hat{e}_{u}-e_{u}\right) \\
& -\sum_{e \in \mathcal{E}_{k}} \int_{e}\left(\left[u_{D}\right]_{A}-\lambda_{R T_{1}}\right)\left[\mathbf{n}_{e} \cdot\left(\hat{e}_{\mathbf{j}}-e_{\mathbf{j}}\right)\right]_{J} d \sigma \\
& \leq\left\|\operatorname{div} e_{\mathbf{j}}\right\|_{0}\left\|\hat{e}_{u}-e_{u}\right\|_{0}+\left\|\left[u_{D}\right]_{A}-\lambda_{R T_{1}}\right\|_{0 ; \hat{\mathcal{E}}_{k}^{-1}}\left\|\left[\mathbf{n}_{e} \cdot\left(\hat{e}_{\mathbf{j}}-e_{\mathbf{j}}\right)\right]_{J}\right\|_{0 ; \hat{\mathcal{E}}_{k}} .
\end{aligned}
$$

Note that the weighted norm $\|\cdot\|_{0 ; \hat{\mathcal{E}}_{k}}$ has the inverse weighting factor compared to $\|\cdot\|_{0 ; \hat{\mathcal{E}}_{k}^{-1}}$ :

$$
\|v\|_{0 ; \hat{\mathcal{E}}_{k}}:=\left(\sum_{e \in \mathcal{E}_{k}} \alpha_{e}^{-1} h_{e}\|v\|_{0 ; e}^{2}\right)^{1 / 2}, \quad v \in L^{2}\left(\mathcal{E}_{k}\right) .
$$


For $\left\|\hat{e}_{u}-e_{u}\right\|_{0}$ we have to establish an adequate upper bound. We obtain

$$
\begin{aligned}
\left\|e_{u}-\hat{e}_{u}\right\|_{0} & \leq \beta_{\operatorname{div}} \sup _{\substack{\mathbf{q} \in R T_{1}\left(\Omega ; \mathcal{T}_{k}\right) \\
\mathbf{q} \neq 0}} \frac{b\left(\mathbf{q}, e_{u}-\hat{e}_{u}\right)}{\|\mathbf{q}\|_{\operatorname{div}}}=\beta_{\operatorname{div}} \sup _{\substack{\mathbf{q} \in R T_{1}\left(\Omega ; \mathcal{T}_{k}\right) \\
\mathbf{q} \neq 0}} \frac{a\left(\mathbf{q}, \hat{e}_{\mathbf{j}}-e_{\mathbf{j}}\right)}{\|\mathbf{q}\|_{\operatorname{div}}} \\
& \leq \alpha_{0}^{-1 / 2} \beta_{\operatorname{div}} a\left(e_{\mathbf{j}}-\hat{e}_{\mathbf{j}}, e_{\mathbf{j}}-\hat{e}_{\mathbf{j}}\right)^{1 / 2} .
\end{aligned}
$$

Due to an inverse estimate for the Raviart-Thomas elements [12, 31], an upper bound for the weighted jump $\left\|\left[\mathbf{n}_{e} \cdot\left(\hat{e}_{\mathbf{j}}-e_{\mathbf{j}}\right)\right]_{J}\right\|_{0 ; \hat{\mathcal{E}}_{k}}$ is given by

$$
\left\|\left[\mathbf{n}_{e} \cdot\left(\hat{e}_{\mathbf{j}}-e_{\mathbf{j}}\right)\right]_{J}\right\|_{0 ; \hat{\varepsilon}_{k}} \leq C_{n o r} a\left(\hat{e}_{\mathbf{j}}-e_{\mathbf{j}}, \hat{e}_{\mathbf{j}}-e_{\mathbf{j}}\right)^{1 / 2},
$$

where the constant $0<C_{\text {nor }}$ is independent of the refinement level. Altogether, we arrive at an upper bound for $a\left(\hat{e}_{\mathbf{j}}, \hat{e}_{\mathbf{j}}\right)^{1 / 2}$ :

$$
a\left(\hat{e}_{\mathbf{j}}, \hat{e}_{\mathbf{j}}\right)^{1 / 2} \leq 2 a\left(e_{\mathbf{j}}, e_{\mathbf{j}}\right)^{1 / 2}+\alpha_{0}^{-1 / 2} \beta_{\operatorname{div}}\left\|\operatorname{div} e_{\mathbf{j}}\right\|_{0}+C_{n o r} \beta_{2 ; \infty}\|\mathbf{j}-\mathbf{j} h\|_{\operatorname{div}} .
$$

It remains to establish an upper bound for $\left\|\operatorname{div} \hat{e}_{\mathbf{j}}\right\|_{0}$. According to the equality $b\left(e_{\mathbf{j}}-\hat{e}_{\mathbf{j}}, v\right)=c\left(e_{u}-\hat{e}_{u}, v\right), v \in W_{1}\left(\Omega ; \mathcal{T}_{k}\right)$, we get

$$
\begin{aligned}
\left\|\operatorname{div} \hat{e}_{\mathbf{j}}\right\|_{0} \leq & \left\|\operatorname{div} e_{\mathbf{j}}\right\|_{0}+\beta_{1}\left\|e_{u}-\hat{e}_{u}\right\|_{0} \\
\leq & \left\|\operatorname{div} e_{\mathbf{j}}\right\|_{0}+\alpha_{0}^{-1 / 2} \beta_{1} \beta_{\text {div }} a\left(\hat{e}_{\mathbf{j}}-e_{\mathbf{j}}, \hat{e}_{\mathbf{j}}-e_{\mathbf{j}}\right)^{1 / 2} \\
\leq & \alpha_{0}^{-1 / 2} \beta_{1} \beta_{\text {div }} a\left(e_{\mathbf{j}}, e_{\mathbf{j}}\right)^{1 / 2}+\left(1+\alpha_{0}^{-1} \beta_{1} \beta_{\text {div }}^{2}\right)\left\|\operatorname{div} e_{\mathbf{j}}\right\|_{0} \\
& +\alpha_{0}^{-1 / 2} \beta_{1} \beta_{\text {div }} C_{\text {nor }} \beta_{2 ; \infty}\|\mathbf{j}-\mathbf{j} h\|_{\text {div }} .
\end{aligned}
$$

To summarize the results, $\left\|\hat{e}_{\mathbf{j}}\right\|_{\text {div }}$ yields a lower bound for $\left\|\mathbf{j}-\mathbf{j}_{h}\right\|_{\text {div }}$ :

$$
C_{a^{-1}}^{e}\left(1+\beta_{\infty}+\frac{C_{a^{-1}}^{0}}{C_{a^{-1}}^{e}}\right)\left\|\mathbf{j}-\mathbf{j}_{h}\right\|_{\operatorname{div}} \geq\left\|\hat{e}_{\mathbf{j}}\right\|_{\operatorname{div}}
$$

where

$$
C_{a^{-1}}^{e}:=\sqrt{2}\left(1+\max \left(1+\frac{\beta_{1} \beta_{\mathrm{div}}}{\sqrt{\alpha_{0}}}, \frac{\beta_{1} \beta_{\mathrm{div}}^{2}}{\alpha_{0}}+\frac{\beta_{\mathrm{div}}}{\sqrt{\alpha_{0}}}\right)\right)
$$

and $C_{a^{-1}}^{0}:=C_{n o r} \beta_{2 ; \infty}\left(1+\alpha_{0}^{-1 / 2} \beta_{1} \beta_{\text {div }}\right)$.

To prove an upper bound for $\left\|\mathbf{j}-\mathbf{j}_{h}\right\|_{\text {div }}$, we again examine $a\left(e_{\mathbf{j}}-\hat{e}_{\mathbf{j}}, e_{\mathbf{j}}-\hat{e}_{\mathbf{j}}\right)$ :

$$
\begin{aligned}
a\left(e_{\mathbf{j}}-\hat{e}_{\mathbf{j}}, e_{\mathbf{j}}\right) & =b\left(e_{\mathbf{j}}, \hat{e}_{u}-e_{u}\right)=c\left(e_{u}, \hat{e}_{u}-e_{u}\right)-\left(f-\Pi_{0} f, \hat{e}_{u}-e_{u}\right)_{0} \\
& =-c\left(\hat{e}_{u}-e_{u}, \hat{e}_{u}-e_{u}\right)+b\left(\hat{e}_{\mathbf{j}}, \hat{e}_{u}-e_{u}\right), \\
a\left(e_{\mathbf{j}}-\hat{e}_{\mathbf{j}}, e_{\mathbf{j}}-\hat{e}_{\mathbf{j}}\right) & \leq-a\left(e_{\mathbf{j}}-\hat{e}_{\mathbf{j}}, \hat{e}_{\mathbf{j}}\right)+\left\|\operatorname{div} \hat{e}_{\mathbf{j}}\right\|_{0}\left\|\hat{e}_{u}-e_{u}\right\|_{0} .
\end{aligned}
$$

Using the upper bound (5.1) for $\left\|e_{u}-\hat{e}_{u}\right\|_{0}$, we obtain

$$
a\left(e_{\mathbf{j}}-\hat{e}_{\mathbf{j}}, e_{\mathbf{j}}-\hat{e}_{\mathbf{j}}\right)^{1 / 2} \leq a\left(\hat{e}_{\mathbf{j}}, \hat{e}_{\mathbf{j}}\right)^{1 / 2}+\alpha_{0}^{-1 / 2} \beta_{\operatorname{div}}\left\|\operatorname{div} \hat{e}_{\mathbf{j}}\right\|_{0}
$$

By means of the triangle inequality we get the upper bound in (2.14) with the constant

$$
C_{\text {loc }}:=1+\sqrt{2}\left(1+\alpha_{0}^{-1} \beta_{1}^{2} \beta_{\text {div }}^{2}\right) \max \left(1, \alpha_{0}^{-1} \beta_{\text {div }}\right) .
$$

The computation of $\left(\hat{e}_{\mathbf{j}}, \hat{e}_{u}\right)$ in $(2.13)$ requires the solution of an $11 \times 11$ saddle point problem for each element. Therefore, the determination of $\hat{e}_{\mathbf{j}}$ is too expensive for a cheap error estimator. The rest of this section is devoted to the reduction of the computational amount. 
As in the previous section, we replace the original bilinear form $\left.a\right|_{T}(\cdot, \cdot)$ by the modified form $\left.\tilde{a}\right|_{T}(\cdot, \cdot)$ and consider three local subproblems. First, it is easy to see that the solution of the subproblem associated with $R T_{0}\left(\Omega ; \mathcal{T}_{k}\right) \times W_{0}\left(\Omega ; \mathcal{T}_{k}\right)$ is equal to zero. Second, let $\left.\hat{e}_{\mathbf{j}_{1}}^{0}\right|_{T} \in \widehat{R T}_{1}^{0}(T)$ be the unique solution of the symmetric, positive definite $3 \times 3$ system

$$
\left.a\right|_{T}\left(\hat{e}_{\mathbf{j}_{1}}^{0}, \mathbf{q}\right)=-\int_{\partial T}\left[u_{D}\right]_{A} \mathbf{n} \cdot \mathbf{q} d \sigma+\left.r\right|_{T}(\mathbf{q}), \mathbf{q} \in \widehat{R T}_{1}^{0}(T) .
$$

We can further reduce the amount of computation, if we replace the stiffness matrix in (5.2) by its spectrally equivalent diagonal matrix. Then, we have to solve one scalar equation for each edge $e_{i} \in \partial T, 1 \leq i \leq 3$ :

$$
\int_{T} \hat{a} \nabla \varphi_{e_{i} ; T} \nabla \Phi_{e_{i}} d x=-\int_{\partial T}\left[u_{D}\right]_{A} \mathbf{n} \cdot\left(\operatorname{curl} \Phi_{e_{i}}\right) d \sigma+\left.r\right|_{T}\left(\operatorname{curl} \Phi_{e_{i}}\right) .
$$

We observe that, due to (4.11),

$$
\left.c_{\text {curl }} a\right|_{T}\left(\hat{e}_{\mathbf{j}_{1}}^{0}, \hat{e}_{\mathbf{j}_{1}}^{0}\right) \leq\left.\sum_{i=1}^{3} a\right|_{T}\left(\operatorname{curl} \varphi_{e_{i}}, \operatorname{curl} \varphi_{e_{i}}\right) \leq\left. C_{\text {curl }} a\right|_{T}\left(\hat{e}_{\mathbf{j}}^{0}, \hat{e}_{\mathbf{j}}^{0}\right) .
$$

Finally, we have to take into account the solution $\left(\left.\hat{e}_{\mathbf{j}_{1}}^{1}\right|_{T},\left.\hat{e}_{u}^{1}\right|_{T}\right) \in \widehat{R T}_{1}^{1}(T) \times \widehat{W}_{1}(T)$, $T \in \mathcal{T}_{k}$, of the following saddle point problem:

$$
\begin{aligned}
\left.a\right|_{T}\left(\hat{e}_{\mathbf{j}_{1}}^{1}, \mathbf{q}\right)+\left.b\right|_{T}\left(\mathbf{q}, \hat{e}_{u_{1}}\right) & =\left.r\right|_{T}(\mathbf{q}), & \mathbf{q} \in \widehat{R T}_{1}^{1}(T), \\
\left.b\right|_{T}\left(\hat{e}_{\mathbf{j}_{1}}^{1}, v\right)-\left.c\right|_{T}\left(\hat{e}_{u_{1}}, v\right) & =-(f, v)_{0 ; T}, & v \in \widehat{W}_{1}(T) .
\end{aligned}
$$

Proof of Theorem 2.4. We only have to show the equivalence of the norms $\left\|\hat{e}_{\mathbf{j}}\right\|_{\text {div }}^{2}$ and $\left\|\hat{e}_{\mathbf{j}_{1}}^{0}\right\|_{\text {div }}^{2}+\left\|\hat{e}_{\mathbf{j}_{1}}^{1}\right\|_{\text {div }}^{2}$. Again, we replace the bilinear form $\left.a\right|_{T}(\cdot, \cdot)$ by $\left.\tilde{a}\right|_{T}(\cdot, \cdot)$. Using the modified residual $\tilde{r}(\mathbf{q}):=r(\mathbf{q})-\sum_{e \in \mathcal{E}_{k}} \int_{e}\left[u_{D}\right]_{A}\left[\mathbf{n}_{e} \cdot \mathbf{q}\right]_{J} d \sigma$, we are in the situation of Theorem 4.1 with $\tilde{r}$ instead of $r$. Then, the same techniques give the assertion.

Remark. An error estimator for the error $\left\|u-u_{h}\right\|_{0}^{2}+\|\mathbf{j}-\mathbf{j} h\|_{\text {div }}^{2}$ is given by

$$
\begin{aligned}
\hat{\eta}_{L}^{2} & :=\sum_{T \in \mathcal{T}_{k}} \hat{\eta}_{L ; T}^{2}, \\
\hat{\eta}_{L ; T}^{2} & :=\eta_{L ; T}^{2}+\left\|\hat{e}_{u_{1}}\right\|_{0 ; T}^{2}, \quad T \in \mathcal{T}_{k} .
\end{aligned}
$$

Since $\left(\hat{e}_{\mathbf{j}}^{1}, \hat{e}_{u}^{1}\right)=\left(\tilde{e}_{\mathbf{j}_{1}}^{1}, \tilde{e}_{u_{1}}\right)$, the assertion is evident.

For the evaluation of the error estimators $\eta_{L}$ and $\hat{\eta}_{L}$ we have to specify the local Dirichlet data $u_{D}$. A possible choice is to take a piecewise quadratic function $v_{\mathbf{j}}$, $\left.v_{\mathbf{j}}\right|_{T} \in P_{2}(T), T \in \mathcal{T}_{k}$. Let $v_{\mathbf{j}}$ be locally defined as the unique quadratic function such that

$$
\left.\nabla v_{\mathbf{j}}\right|_{T}=-\left.a^{-1} \tilde{\mathbf{j}}_{h}\right|_{T},\left.\quad \Pi_{0} v_{\mathbf{j}}\right|_{T}=\left.\tilde{u}_{h}\right|_{T}, \quad T \in \mathcal{T}_{k},
$$

and take $u_{D}=v_{\mathbf{j}}$. In subsection 7.2, we will see that this definition guarantees the local equivalence between the hierarchical error estimator and the estimator based on the solution on local subproblems. 


\section{ERror ESTIMATOR BASED ON A SUPERCONVERGENCE RESUlT}

The starting point for the construction of the error estimator is the following discrete nonconforming variational problem:

$$
a_{N}\left(\Psi_{N C}, \eta\right)=\left(\Pi_{0} f, \eta\right)_{0}, \quad \eta \in N\left(\Omega ; \mathcal{T}_{k}\right),
$$

where the bilinear form $a_{N}: N\left(\Omega ; \mathcal{T}_{k}\right) \times N\left(\Omega ; \mathcal{T}_{k}\right) \rightarrow \mathbb{R}$ is given by

$$
a_{N}(\psi, \eta):=\sum_{T \in \mathcal{T}_{k}} \int_{T}\left(\hat{P}_{a^{-1}}(a \nabla \psi) \cdot \nabla \eta+b \Pi_{0} \psi \cdot \Pi_{0} \eta\right) d x, \quad \psi, \eta \in N\left(\Omega ; \mathcal{T}_{k}\right),
$$

and the nonconforming ansatz space $N\left(\Omega ; \mathcal{T}_{k}\right)$ is the lowest order Crouzeix-Raviart space augmented by cubic bubble functions:

$$
N\left(\Omega ; \mathcal{T}_{k}\right):=C R\left(\Omega ; \mathcal{T}_{k}\right) \oplus B\left(\Omega ; \mathcal{T}_{k}\right) .
$$

Here, $C R\left(\Omega ; \mathcal{T}_{k}\right):=\left\{v \in L^{2}(\Omega)|v|_{T} \in P_{1}(T), T \in \mathcal{T}_{k},\left.v\right|_{T_{1}}\left(m_{e}\right)=\left.v\right|_{T_{2}}\left(m_{e}\right), \partial T_{1} \cap\right.$ $\left.\partial T_{2}=e \subset \Omega,\left.v\right|_{T}\left(m_{e}\right)=0, e \subset \partial T \cap \partial \Omega\right\}$ and $B\left(\Omega ; \mathcal{T}_{k}\right):=\left\{v \in L^{2}(\Omega)|v|_{T} \in\right.$ $\left.P_{3}(T),\left.v\right|_{\partial T}=0, T \in \mathcal{T}_{k}\right\}$. The local operator $\Pi_{0}$ is the $L^{2}$-projection onto $W_{0}\left(\Omega ; \mathcal{T}_{k}\right)$, whereas $\hat{P}_{a^{-1}}$ denotes the orthogonal projection onto $R T_{0}^{-1}\left(\Omega ; \mathcal{T}_{k}\right)$ with respect to the bilinear form $a(\cdot, \cdot)$. Then, the following equalities are true $[2,12]$ :

$$
u_{h}=\Pi_{0} \Psi_{N C}, \quad \lambda_{h}=\Pi_{e} \Psi_{N C}, \quad \mathbf{j}_{h}=-\hat{P}_{a^{-1}}\left(a \nabla \Psi_{N C}\right),
$$

where $\left(\mathbf{j}_{h}, u_{h}, \lambda_{h}\right) \in R T_{0}^{-1}\left(\Omega ; \mathcal{T}_{k}\right) \times W_{0}\left(\Omega ; \mathcal{T}_{k}\right) \times M_{0}\left(\Omega ; \mathcal{E}_{k}\right)$ denotes the unique solution of the mixed hybrid formulation of the variational problem (1.4):

$$
\begin{aligned}
a\left(\mathbf{j}_{h}, \mathbf{q}\right)+b\left(\mathbf{q}, u_{h}\right)+d\left(\lambda_{h}, \mathbf{q}\right) & =0, & & \mathbf{q} \in R T_{0}^{-1}\left(\Omega ; \mathcal{T}_{k}\right), \\
b\left(\mathbf{j}_{h}, v\right)-c\left(u_{h}, v\right) & =-(f, v)_{0}, & & v \in W_{0}\left(\Omega ; \mathcal{T}_{k}\right), \\
d\left(\mu, \mathbf{j}_{h}\right) & =0, & & \mu \in M_{0}\left(\Omega ; \mathcal{E}_{k}\right) .
\end{aligned}
$$

Here, $M_{0}\left(\Omega ; \mathcal{E}_{k}\right)$ is the ansatz space for the Lagrange multipliers

$$
M_{0}\left(\Omega ; \mathcal{E}_{k}\right):=\left\{\mu \in L^{2}\left(\mathcal{E}_{k}\right)|\mu|_{e} \in P_{0}(e), e \in \mathcal{E}_{k},\left.\mu\right|_{e}=0, e \subset \partial \Omega\right\}
$$

and $\Pi_{e}$ stands for the $L^{2}$-projection onto $M_{0}\left(\Omega ; \mathcal{E}_{k}\right)$. Finally, the bilinear form $d_{R T}: M_{0}\left(\Omega ; \mathcal{E}_{k}\right) \times R T_{0}^{-1}\left(\Omega ; \mathcal{T}_{k}\right) \rightarrow \mathbb{R}$ is given by

$$
d(\mu, \mathbf{q}):=\sum_{T \in \mathcal{T}_{k}} \int_{\partial T} \mu \mathbf{n} \cdot \mathbf{q} d \sigma, \quad \mu \in M_{0}\left(\Omega ; \mathcal{E}_{k}\right), \mathbf{q} \in R T_{0}^{-1}\left(\Omega ; \mathcal{T}_{k}\right) .
$$

Now, we assume the existence of a constant $0 \leq \beta<1$ such that

$$
\left\|u-\hat{u}_{R T_{0}}\right\|_{0} \leq \beta\left\|u-u_{h}\right\|_{0},
$$

with $\hat{u}_{R T_{0}} \in C R\left(\Omega ; \mathcal{T}_{k}\right)$ being the nonconforming extension of $\lambda_{h}$. This saturation assumption is motivated by a superconvergence result that holds true in the case of mixed hybridization, stating that the nonconforming extension $\hat{u}_{R T_{0}}$ of the multiplier $\lambda_{h}$ does provide a better approximation of the primal variable $u$ than the piecewise constant approximation $u_{h}$ (see $[2,12]$ ). It is easy to see that $(6.4)$ gives rise to an upper and a lower bound for the discretization error of the primal variable $u$ in the $L^{2}$-norm:

$$
(1+\beta)^{-1}\left\|\hat{u}_{R T_{0}}-u_{h}\right\|_{0} \leq\left\|u-u_{h}\right\|_{0} \leq(1-\beta)^{-1}\left\|\hat{u}_{R T_{0}}-u_{h}\right\|_{0} .
$$

Up to now, the error estimator depends on $u_{h}$ and $\hat{u}_{R T_{0}}$. If the original system (1.4) is solved, the nonconforming approximation $\hat{u}_{R T_{0}}$ is not available without additional computation. We have to solve additional local problems to obtain $\hat{u}_{R T_{0}}$. 
In the rest of this section, the equivalence between $\left\|u_{h}-\hat{u}_{R T_{0}}\right\|_{0}^{2}$ and a weighted sum of the squared jumps of $u_{h}$ across the edges $e \in \mathcal{E}_{k}$ will be established.

Theorem 6.1. Let $\left(\mathbf{j}_{h}, u_{h}, \lambda_{h}\right) \in R T_{0}^{-1}\left(\Omega ; \mathcal{T}_{k}\right) \times W_{0}\left(\Omega ; \mathcal{T}_{k}\right) \times M_{0}\left(\Omega ; \mathcal{E}_{k}\right)$ be the unique solution of (6.3), and assume that $\hat{u}_{R T_{0}} \in C R\left(\Omega ; \mathcal{T}_{k}\right)$ is the nonconforming extension of $\lambda_{h}$. Then, there exist constants $0<\sigma_{0} \leq \sigma_{1}$, depending only on the shape regularity of $\mathcal{T}_{k}$ and the ellipticity constants in (1.2), such that

$$
\sigma_{0}\left(\sum_{e^{\prime} \in \mathcal{E}_{k}} h_{e}^{2}\left(\left.\left[u_{h}\right]_{J}\right|_{e}\right)^{2}\right)^{1 / 2} \leq\left\|u_{h}-\hat{u}_{R T_{0}}\right\|_{0} \leq \sigma_{1}\left(\sum_{e^{\prime} \in \mathcal{E}_{k}} h_{e}^{2}\left(\left.\left[u_{h}\right]_{J}\right|_{e}\right)^{2}\right)^{1 / 2} .
$$

Proof. A detailed proof of the theorem can be found in [19, Thm. 4.1], [32, Thm. 5.8]. Here, we will only sketch the main ideas. By straightforward computation, we obtain

$$
c\left\|u_{h}-\hat{u}_{R T_{0}}\right\|_{0}^{2} \leq \sum_{e \in \mathcal{E}_{k}} h_{e}^{2}\left(2\left(\left.\left[u_{h}\right]_{A}\right|_{e}-\left.\lambda_{h}\right|_{e}\right)^{2}+\frac{1}{2}\left(\left.\left[u_{h}\right]_{J}\right|_{e}\right)^{2}\right) \leq C\left\|u_{h}-\hat{u}_{R T_{0}}\right\|_{0}^{2},
$$

where the constants $0<c \leq C$ are independent of the refinement level. As a direct consequence of (6.6), we obtain the lower bound in (6.5).

However, the proof of the upper bound is more involved. It is sufficient to show that

$$
\sum_{e \in \mathcal{E}_{k}} h_{e}^{2}\left(\left.\left(\left[u_{h}\right]_{A}-\lambda_{h}\right)\right|_{e}\right)^{2} \leq c \sum_{e \in \mathcal{E}_{k}} h_{e}^{2}\left(\left.\left[u_{h}\right]_{J}\right|_{e}\right)^{2}
$$

with an appropriate positive constant $c$. As a first step, one can establish the following relationship between $\lambda_{h}$ and the averages and jumps of $u_{h}$ :

$$
\left.\lambda_{h}\right|_{e}=\left.\left[u_{h}\right]_{A}\right|_{e}-\left.\left.\sum_{e^{\prime} \in \mathcal{E}_{k}} \frac{h_{e^{\prime}}}{h_{e}}\left[u_{h}\right]_{J}\right|_{e^{\prime}}\left[\mathbf{n}_{e^{\prime}} \cdot P_{a^{-1}}\left(\boldsymbol{\tau}_{e}\right)\right]_{A}\right|_{e^{\prime}}
$$

where $P_{a^{-1}}$ denotes the global orthogonal projection onto $R T_{0}\left(\Omega ; \mathcal{T}_{k}\right)$ with respect to the weighted $L^{2}$-inner product $a(\cdot, \cdot)$, and $\boldsymbol{\tau}_{e}$ stands for a local function of $R T_{0}^{-1}\left(\Omega ; \mathcal{T}_{k}\right)$ associated with the edge $e$,

$$
\boldsymbol{\tau}_{e}=\frac{1}{2}\left(\boldsymbol{\tau}_{e}^{T_{i n}}+\boldsymbol{\tau}_{e}^{T_{o u t}}\right) .
$$

Here, $\boldsymbol{\tau}_{e}^{T_{\text {in }}}, \boldsymbol{\tau}_{e}^{T_{\text {out }}}$ are the nodal basis vector fields in $R T_{0}^{-1}\left(\Omega ; \mathcal{T}_{k}\right)$ with support in $T_{\text {in }}$ and $T_{\text {out }}$, respectively, given by

$$
\left.\mathbf{n} \cdot \boldsymbol{\tau}_{e}^{T}\right|_{e^{\prime}}=\delta_{e, e^{\prime}}, \quad e^{\prime} \subset \partial T, T \in\left\{T_{\text {in }}, T_{\text {out }}\right\} .
$$

Note that $\boldsymbol{\tau}_{e}$ is not contained in $H(\operatorname{div} ; \Omega)$ for an interior edge. For the next step, one has to consider the projection $P_{a^{-1}}$ in more detail and to prove that the spectral radius of $P_{a^{-1}} P_{a^{-1}}^{T}$ is bounded independently of the refinement level. This can be achieved by considering the local matrix representations of the positive definite operator $A$ associated with the bilinear form $a(\cdot, \cdot)$ and the natural embedding of $R T_{0}\left(\Omega ; \mathcal{T}_{k}\right)$ into $R T_{0}^{-1}\left(\Omega ; \mathcal{T}_{k}\right)$.

Proof of Theorem 2.5. It is easy to see that

$$
\left|h_{e}\right|\left\|[w]_{J}\right\|_{0 ; e}^{2} \leq \frac{2}{\kappa_{0}}\|w\|_{0 ; T_{1} \cup T_{2}}^{2}, \quad T_{1} \cap T_{2}=e, w \in W_{0}\left(\Omega ; \mathcal{T}_{k}\right) .
$$


Due to the triangle inequality and Theorem 2.5 we obtain

$$
\begin{aligned}
& \left\|u-\tilde{u}_{h}\right\|_{0} \leq \frac{\sigma_{1}}{1-\beta}\left(\sum_{e^{\prime} \in \mathcal{E}_{k}} h_{e}\left\|\left[\tilde{u}_{h}\right]_{J}\right\|_{0 ; e}^{2}\right)^{1 / 2}+\left(\frac{\sigma_{1}}{1-\beta} \sqrt{6 \kappa_{0}^{-1}}+1\right)\left\|u_{h}-\tilde{u}_{h}\right\|_{0}, \\
& \left\|u-\tilde{u}_{h}\right\|_{0} \geq \frac{\sigma_{0}}{1+\beta}\left(\sum_{e^{\prime} \in \mathcal{E}_{k}} h_{e}\left\|\left[\tilde{u}_{h}\right]_{J}\right\|_{0 ; e}^{2}\right)^{1 / 2}-\left(\frac{\sigma_{0}}{1+\beta} \sqrt{6 \kappa_{0}^{-1}}+1\right)\left\|u_{h}-\tilde{u}_{h}\right\|_{0},
\end{aligned}
$$

and hence the assertion is proved.

\section{Comparison of the DifFerent error estimators}

The error estimators $\hat{\eta}_{R}, \eta_{H}, \hat{\eta}_{H}, \eta_{L}, \hat{\eta}_{L}, \eta_{S}$ and $\hat{\eta}_{S}$ have been investigated independently in Sections 3-6, respectively. In this section, we examine the relationships between their local contributions. For simplicity we restrict ourselves to the Poisson equation with homogeneous Dirichlet boundary data

$$
-\Delta u=f \quad \text { in } \Omega \quad \text { and } \quad u=0 \quad \text { on } \partial \Omega,
$$

and we assume that the exact discrete solution is available.

\subsection{Equivalence between the residual based and the hierarchical error estimator.}

Theorem 7.1. Let $\hat{\eta}_{R ; T}$ and $\hat{\eta}_{H ; T}, T \in \mathcal{T}_{k}$, be given by (2.2) and (4.12), respectively. Then, there exist constants $c_{R ; H}, C_{R ; H}$ and $\Gamma_{R ; H}>0$, independent of the refinement level and $T \in \mathcal{T}_{k}$, such that

$$
\begin{aligned}
c_{R ; H} \hat{\eta}_{H ; T}^{2} \leq \hat{\eta}_{R ; T}^{2} \leq & C_{R ; H} \hat{\eta}_{H ; T}^{2}+\left\|f-\Pi_{1} f\right\|_{0 ; T}^{2} \\
& +\Gamma_{R ; H} h_{T}^{2}\left(h_{T}^{2}\left\|\Pi_{0} f\right\|_{0 ; T}^{2}+\sum_{i=1}^{3} h_{e_{i}}\left\|\left[\Pi_{0} f\right]_{J}\right\|_{0 ; e_{i}}^{2}\right) .
\end{aligned}
$$

Proof. We recall that the local components of $\hat{\eta}_{R}$ and $\hat{\eta}_{H}$ are given by

$$
\begin{aligned}
& \hat{\eta}_{R ; T}^{2}:=\left\|f-\Pi_{0} f\right\|_{0 ; T}^{2}+h_{T}^{2}\left\|a^{-1} \tilde{\mathbf{j}}_{h}\right\|_{0 ; T}^{2}+\sum_{i=1}^{3} w_{i} \alpha_{e_{i}} h_{e_{i}}\left\|\left[a^{-1} \tilde{\mathbf{j}}_{h} \cdot \mathbf{t}_{e_{i}}\right]_{J}\right\|_{0 ; e_{i}}^{2}, \\
& \hat{\eta}_{H ; T}^{2}:=\left\|\left.\tilde{\mathbf{j}}_{\mathbf{j}_{1}}^{1}\right|_{T}\right\|_{\text {div }}^{2}+\sum_{i=1}^{3} w_{i}\left\|\operatorname{curl} \varphi_{e_{i}}\right\|_{\text {div }}^{2}+\left\|\tilde{e}_{u_{1}}\right\|_{0 ; T}^{2}, \quad T \in \mathcal{T}_{k} .
\end{aligned}
$$

In order to establish their equivalence we proceed in several steps. First, we determine an upper bound for $\hat{\eta}_{H ; T}$. To this end we consider the three parts of $\hat{\eta}_{H ; T}$ separately. It is easy to see that there exists a constant $c_{1}>0$, independent of the refinement level, such that

$$
\|\mathbf{q}\|_{0 ; T} \leq c_{1} h_{T}\|\operatorname{div} \mathbf{q}\|_{0 ; T}, \quad \mathbf{q} \in \widehat{R T}_{1}^{1}(T), \quad T \in \mathcal{T}_{k} .
$$

Inequality (7.2) guarantees an upper bound for $\left\|\left.\tilde{e}_{\mathbf{j}_{1}}^{1}\right|_{T}\right\|_{\text {div }}^{2}$ :

$$
\begin{aligned}
\left\|\left.\tilde{e}_{\mathbf{j}_{1}}^{1}\right|_{T}\right\|_{\text {div }}^{2} & =\left\|\tilde{e}_{\mathbf{j}_{1}}^{1}\right\|_{0 ; T}^{2}+\left\|\operatorname{div} \tilde{e}_{\mathbf{j}_{1}}^{1}\right\|_{0 ; T}^{2} \\
& \leq\left(1+c_{1}^{2} h_{T}^{2}\right)\left\|\operatorname{div} \tilde{e}_{\mathbf{j}_{1}}^{1}\right\|_{0 ; T}^{2} \leq\left(1+c_{1}^{2} h_{T}^{2}\right)\left\|f-\Pi_{0} f\right\|_{0 ; T}^{2} .
\end{aligned}
$$


On the other hand, an upper bound for the second term $\left\|\operatorname{curl} \varphi_{e_{i}}\right\|_{\text {div }}$ is obtained by

$$
\begin{aligned}
& \left\|\operatorname{curl} \varphi_{e_{i}}\right\|_{\operatorname{div}}=\sup _{\substack{\left.\mathbf{q} \in \widehat{R T} T_{1}^{0}\left(\Omega ; \mathcal{T}_{k}\right) \\
\mathbf{q} \cdot \mathbf{n}\right|_{e}=0, e \neq e_{i}}} \frac{\int_{\Omega} \operatorname{curl} \varphi_{e_{i}} \cdot \mathbf{q} d x}{\|\mathbf{q}\|_{0}}=\sup _{\substack{\left.\mathbf{q} \in \widehat{R T}\left(1, \mathcal{T}_{k}\right) \\
\mathbf{q n}\right|_{e}=0, e \neq e_{i}}} \frac{r(\mathbf{q})}{\|\mathbf{q}\|_{0}} \\
& \leq\left(\int_{e_{i}}\left[\mathbf{j}_{h} \cdot \mathbf{t}_{e_{i}}\right]_{J}^{2} d \sigma\right)^{1 / 2} \cdot\left\|\nabla \Phi_{e_{i}}\right\|_{0}^{-1}\left(\int_{e_{i}} \Phi_{e_{i}}^{2} d \sigma\right)^{1 / 2} \\
& \leq c_{2} h_{e_{i}}^{1 / 2}\left\|\left[\mathbf{j}_{h} \cdot \mathbf{t}_{e_{i}}\right]_{J}\right\|_{0 ; e_{i}},
\end{aligned}
$$

where the constant $c_{2}$ depends only on the geometry of $\mathcal{T}_{0}$. For an estimation of the third term, we note that $\left.\tilde{e}_{u_{1}}\right|_{T}$ is an element of $\widehat{W}_{1}(T)$. Hence

$$
\begin{aligned}
\left\|\tilde{e}_{u_{1}}\right\|_{0 ; T} & =\sup _{\substack{\mathbf{q} \in \widehat{R T}_{1}^{1}(T) \\
\mathbf{q} \neq 0}} \frac{\int_{T} \operatorname{div} \mathbf{q} \tilde{e}_{u_{1}} d x}{\|\operatorname{div} \mathbf{q}\|_{0 ; T}}=\sup _{\substack{\mathbf{q} \in \widehat{R T}_{1}^{1}(T) \\
\mathbf{q} \neq 0}} \frac{\int_{T}\left(\tilde{e}_{\mathbf{j}_{1}}^{1}+\mathbf{j}_{h}\right) \cdot \mathbf{q} d x}{\|\operatorname{div} \mathbf{q}\|_{0 ; T}} \\
& \leq c_{1} h_{T}\left(\left\|\tilde{e}_{\mathbf{j}_{1}}^{1}\right\|_{0 ; T}+\left\|\mathbf{j}_{h}\right\|_{0 ; T}\right) .
\end{aligned}
$$

The right-hand sides in (7.3), (7.4) and (7.5) give rise to $\hat{\eta}_{R ; T}^{2}$. Thus, we have derived an upper bound for $\hat{\eta}_{H ; T}$, which in turn implies a lower bound for $\hat{\eta}_{R ; T}$.

It remains to establish the upper bound for $\hat{\eta}_{R ; T}^{2}$. Again, we consider the different parts of $\hat{\eta}_{R ; T}^{2}$ separately. As a first step, we examine $h_{T}^{2}\left\|\mathbf{j}_{h}\right\|_{0 ; T}^{2}$. Let $\mathbf{j}_{d} \in R T_{0}(T)$ be given by

$$
\mathbf{j}_{d}=\frac{\Pi_{0} f}{2}\left(\mathbf{x}-\mathbf{x}_{c}\right)
$$

where $\mathbf{x}_{c}$ denotes the center of gravity of $T$. Then, it is easy to see that

$$
\operatorname{div}\left(\left.\mathbf{j}_{h}\right|_{T}-\mathbf{j}_{d}\right)=0 \text {. }
$$

Note that there exists a unique $v \in P_{1}(T)$ such that $\Pi_{0} v=0$ and $\nabla v=\left.\mathbf{j}_{h}\right|_{T}-\mathbf{j}_{d}$.

Further, let $\mathbf{p} \in \widehat{R T}_{1}^{1}(T)$ be uniquely given by means of $-\operatorname{div} \mathbf{p}=v$. There exists a constant $c_{3}$, independent of the refinement level and $T \in \mathcal{T}_{k}$, such that $\|w\|_{0 ; T} \geq c_{3} h_{T}|w|_{1 ; T}$ for all $w \in P_{1}(T)$. Then, we obtain

$$
c_{3} h_{T}\left\|\left.\mathbf{j}_{h}\right|_{T}-\mathbf{j}_{d}\right\|_{0 ; T}\|\operatorname{div} \mathbf{p}\|_{0 ; T} \leq\|v\|_{0 ; T}^{2}=-\int_{T} \operatorname{div} \mathbf{p} v d x=\int_{T}\left(\left.\mathbf{j}_{h}\right|_{T}-\mathbf{j}_{d}\right) \cdot \mathbf{p} d x
$$

whence

$$
\begin{aligned}
\left\|\mathbf{j}_{h}-\mathbf{j}_{d}\right\|_{0 ; T} & \leq\left(c_{3} h_{T}\right)^{-1} \sup _{\mathbf{q} \in \overparen{R T} 1(T)} \int_{T}\left(\mathbf{j}_{h}-\mathbf{j}_{d}\right) \cdot \mathbf{q} d x\|\operatorname{div} \mathbf{q}\|_{0 ; T}^{-1} \\
& \leq c_{1} c_{3}^{-1}\left(\left\|\mathbf{j}_{d}\right\|_{0 ; T}+\left\|\tilde{e}_{\mathbf{j}_{1}}^{1}\right\|_{0 ; T}\right)+\left(c_{3} h_{T}\right)^{-1}\left\|\tilde{e}_{u_{1}}\right\|_{0 ; T} .
\end{aligned}
$$

On the other hand, we get

$$
\left\|\mathbf{j}_{d}\right\|_{0 ; T} \leq \frac{1}{2} h_{T}\left\|\Pi_{0} f\right\|_{0 ; T}
$$


Altogether, this results in an upper bound for $h_{T}^{2}\left\|\mathbf{j}_{h}\right\|_{0 ; T}^{2}$ :

$$
h_{T}^{2}\left\|\mathbf{j}_{h}\right\|_{0 ; T}^{2} \leq 3\left(\frac{1}{c_{3}}\right)^{2}\left\|\tilde{e}_{u_{1}}\right\|_{0 ; T}^{2}+3\left(\frac{c_{1}}{c_{3}}\right)^{2} h_{T}^{2}\left\|\tilde{e}_{\mathbf{j}_{1}}^{1}\right\|_{0 ; T}^{2}+\frac{3}{4}\left(1+\frac{c_{1}}{c_{3}}\right)^{2} h_{T}^{4}\left\|\Pi_{0} f\right\|_{0 ; T}^{2} \text {. }
$$

Finally, we consider $\left\|\left[\mathbf{j}_{h} \cdot \mathbf{t}_{e}\right]_{J}\right\|_{0 ; e}$ and remark that $\left[\mathbf{j}_{h} \cdot \mathbf{t}_{e}\right]_{J} \in P_{1}(e)$ can be written as

$$
\left[\mathbf{j}_{h} \cdot \mathbf{t}_{e}\right]_{J}\left(\mathbf{x}_{s}\right)=a_{e}+b_{e}\left(s-h_{e} / 2\right), \quad s \in\left[0 ; h_{e}\right], \quad \mathbf{x}_{s}:=\mathbf{p}_{e}+s \cdot \mathbf{t}_{e},
$$

where $a_{e}:=\left[\mathbf{j}_{h} \cdot \mathbf{t}_{e}\right]_{J}\left(m_{e}\right)$ and $b_{e}:=\left(d\left[\mathbf{j}_{h} \cdot \mathbf{t}_{e}\right]_{J} / d s\right)\left(m_{e}\right)$. Here, $m_{e}$ denotes the midpoint of the edge $e$ and $\mathbf{p}_{e}$ is that vertex of $e$ such that the other vertex is given by $\mathbf{p}_{e}+h_{e} \mathbf{t}_{e}$. Since $b_{e}=\nabla\left[\mathbf{j}_{h} \cdot \mathbf{t}_{e}\right]_{J} \cdot \mathbf{t}_{e}$, we get

$$
b_{e}=\frac{1}{2}\left[\Pi_{0} f\right]_{J},
$$

whence

$$
h_{e}\left\|\left[\mathbf{j}_{h} \cdot \mathbf{t}_{e}\right]_{J}\right\|_{0 ; e}^{2}=a_{e}^{2} h_{e}^{2}+\frac{1}{12} h_{e}^{3}\left\|\left[\Pi_{0} f\right]_{J}\right\|_{0 ; e}^{2} .
$$

On the other hand,

$$
\begin{aligned}
\pm a_{e} & = \pm 3\left(2 h_{e}\right)^{-1} \int_{e}\left[\mathbf{j}_{h} \cdot \mathbf{t}_{e}\right]_{J} \Phi_{e} d \sigma= \pm 3\left(2 h_{e}\right)^{-1} r\left(\operatorname{curl} \Phi_{e}\right) \\
& \leq 3\left(2 h_{e}\right)^{-1}\left|\Phi_{e}\right|_{1}\left\|\operatorname{curl} \varphi_{e}\right\|_{\text {div }} \leq 3\left(2 h_{e}\right)^{-1} c_{4}\left\|\operatorname{curl} \varphi_{e}\right\|_{\text {div }} .
\end{aligned}
$$

Note that in view of

$$
0<\hat{c}_{4} \leq\left|\Phi_{e}\right|_{1} \leq c_{4}, \quad e \in \mathcal{E}_{k}, k \geq 0,
$$

the positive constant $c_{4}>0$ depends only on the geometry of $\mathcal{T}_{0}$. We thus get

$$
h_{e}\left\|\left[\mathbf{j}_{h} \cdot \mathbf{t}_{e}\right]_{J}\right\|_{0 ; e}^{2} \leq \frac{9}{4} c_{4}^{2}\left\|\operatorname{curl} \varphi_{e}\right\|_{\text {div }}^{2}+\frac{1}{12} h_{e}^{3}\left\|\left[\Pi_{0} f\right]_{J}\right\|_{0 ; e}^{2} .
$$

Summarizing the preceding results, we establish an upper bound for $\hat{\eta}_{R ; T}^{2}$ :

$$
\begin{gathered}
\hat{\eta}_{R ; T}^{2} \leq \max \left(1,3 c_{1}^{2} c_{3}^{-2} h_{T}^{2}\right)\left\|\left.\tilde{e}_{\mathbf{j}_{1}}^{1}\right|_{T}\right\|_{\text {div }}^{2}+\frac{9}{4} c_{4}^{2} \sum_{i=1}^{3} w_{i}\left\|\operatorname{curl} \varphi_{e_{i}}\right\|_{\text {div }}^{2}+3 c_{3}^{-2}\left\|\tilde{e}_{u_{1}}\right\|_{0 ; T}^{2} \\
\quad+\frac{3}{4}\left(1+c_{1} c_{3}^{-1}\right)^{2} h_{T}^{4}\left\|\Pi_{0} f\right\|_{0 ; T}^{2}+\frac{1}{12} \sum_{i=1}^{3} w_{i} h_{e_{i}}^{3}\left\|\left[\Pi_{0} f\right]_{J}\right\|_{0 ; e_{i}}^{2}+\left\|f-\Pi_{1} f\right\|_{0 ; T}^{2}
\end{gathered}
$$

The fourth, fifth and sixth terms in the upper bound of $\hat{\eta}_{R ; T}$ are, in general, higher order perturbations of $\hat{\eta}_{R ; T}$. Provided the grid size is small enough, they can be neglected.

7.2. Remarks on the error estimator based on the solution of local subproblems. We will show that the estimators $\eta_{L}$ and $\hat{\eta}_{L}$ based on the solution of local subproblems and the hierarchical basis error estimators $\eta_{H}$ and $\hat{\eta}_{H}$ are locally equivalent.

Theorem 7.2. Let $\eta_{H ; T}, \eta_{L ; T}$ and $\hat{\eta}_{H ; T}, \hat{\eta}_{H ; T}, T \in \mathcal{T}_{k}$, be given by (2.10), (2.15), (4.12) and (5.5), respectively. Then

$$
\eta_{H ; T}^{2} \leq \eta_{L ; T}^{2}+\frac{1}{2} \sum_{e=\partial T \cap \partial T_{e}} \eta_{L ; T_{e}}^{2}, \quad \hat{\eta}_{H ; T}^{2} \leq \hat{\eta}_{L ; T}^{2}+\frac{1}{2} \sum_{e=\partial T \cap \partial T_{e}} \hat{\eta}_{L ; T_{e}}^{2} .
$$


where $e=\partial T \cap \partial T_{e}$ denotes an interior edge and $T_{e}$ stands for the adjacent element sharing the edge e with $T$.

Moreover, if we choose $u_{D}=v_{\mathbf{j}}$ with $\nabla v_{\mathbf{j}}=\mathbf{j}_{h}$ and $\left[v_{\mathbf{j}}\right]_{A}=0,\left[\mathbf{t}_{e} \cdot \mathbf{j}_{h}\right]_{J}=0$ for $e \subset \partial \Omega$ in (5.3), then there exist constants $C_{L ; H}, \hat{C}_{L ; H}>0$, independent of the refinement level and $T \in \mathcal{T}_{k}$, such that

$$
\eta_{L ; T}^{2} \leq C_{L ; H} \eta_{H ; T}, \quad \hat{\eta}_{L ; T}^{2} \leq \hat{C}_{L ; H} \hat{\eta}_{H ; T}
$$

Proof. The local contributions of $\eta_{H}, \hat{\eta}_{H}$ and $\eta_{L}, \hat{\eta}_{L}$ are given by

$$
\begin{aligned}
& \eta_{H ; T}^{2}=\left\|\tilde{e}_{\mathbf{j}_{1}}^{1}\right\|_{\text {div } ; T}^{2}+\sum_{i=1}^{3} w_{i}\left\|\operatorname{curl} \varphi_{e_{i}}\right\|_{\text {div }}^{2}, \quad \hat{\eta}_{H ; T}^{2}=\eta_{H ; T}^{2}+\| \tilde{e}_{u_{1} \|_{0 ; T}}, \\
& \eta_{L ; T}^{2}=\left\|\hat{e}_{\mathbf{j}_{1}}^{1}\right\|_{\text {div } ; T}^{2}+\left.\sum_{i=1}^{3} a\right|_{T}\left(\operatorname{curl} \varphi_{e_{i} ; T}, \operatorname{curl} \varphi_{e_{i} ; T}\right), \quad \hat{\eta}_{L ; T}^{2}=\eta_{L ; T}^{2}+\left\|\hat{e}_{u_{1}}\right\|_{0 ; T},
\end{aligned}
$$

where $w_{i}=1$ if $e_{i} \subset \partial \Omega$ and $w_{i}=\frac{1}{2}$ if $e_{i}$ is an interior edge.

We note that $\left(\hat{e}_{\mathbf{j}_{1}}^{1}, \hat{e}_{u_{1}}\right)=\left(\tilde{e}_{\mathbf{j}_{1}}^{1}, \tilde{e}_{u_{1}}\right)$ and $\varphi_{\hat{e}_{\mathbf{j}}^{0}}^{e}=\varphi_{\tilde{e}_{\mathbf{j}_{1}}}^{e}$ if $e \subset \partial \Omega$. On the other hand, for an interior edge $e=\partial T \cap \partial T_{e}$ we get

$$
\begin{aligned}
a\left(\operatorname{curl} \varphi_{e}, \operatorname{curl} \varphi_{e}\right) & =r\left(\operatorname{curl} \varphi_{e}\right) \\
& =\left.a\right|_{T}\left(\operatorname{curl} \varphi_{e ; T}, \operatorname{curl} \varphi_{e}\right)+\left.a\right|_{T_{e}}\left(\operatorname{curl} \varphi_{e ; T}, \operatorname{curl} \varphi_{e}\right) .
\end{aligned}
$$

Thanks to Young's inequality, this proves (7.7).

For the proof of (7.8), we choose $u_{D}=v_{\mathbf{j}}$ in (5.3) and obtain

$$
\begin{aligned}
& \int_{T} \nabla \varphi_{e ; T} \cdot \nabla \Phi_{e} d x=\int_{\partial T}\left[v_{\mathbf{j}}\right]_{A} \nabla \Phi_{e} \cdot \mathbf{t} d \sigma-\left.a\right|_{T}\left(\mathbf{j}_{h}, \operatorname{curl} \Phi_{e}\right) \\
&=\int_{\partial T} \mathbf{j}_{h} \cdot \mathbf{t} \Phi_{e} d \sigma-\int_{\partial T}\left[\nabla v_{\mathbf{j}} \cdot \mathbf{t}\right]_{A} \Phi_{e} d \sigma= \pm \frac{1}{2} \int_{e}\left[\mathbf{j}_{h} \cdot \mathbf{t}_{e}\right]_{J} \Phi_{e} d \sigma
\end{aligned}
$$

for an interior edge. Consequently

$$
\begin{aligned}
\left.\left.a\right|_{T}\left(\operatorname{curl} \Phi_{e}, \operatorname{curl} \Phi_{e}\right) a\right|_{T}\left(\operatorname{curl} \varphi_{e ; T}, \operatorname{curl} \varphi_{e ; T}\right)=\frac{1}{4}\left(\int_{e}\left[\mathbf{t} \cdot \mathbf{j}_{h}\right]_{J} \Phi_{e} d \sigma\right)^{2} \\
\quad=\frac{1}{4} a\left(\operatorname{curl} \varphi_{e}, \operatorname{curl} \Phi_{e}\right)^{2}=\frac{1}{4} a\left(\operatorname{curl} \varphi_{e}, \operatorname{curl} \varphi_{e}\right) a\left(\operatorname{curl} \Phi_{e}, \operatorname{curl} \Phi_{e}\right) \\
\quad \leq\left.\frac{1}{4}(1+C) a\left(\operatorname{curl} \varphi_{e}, \operatorname{curl} \varphi_{e}\right) a\right|_{T}\left(\operatorname{curl} \Phi_{e}, \operatorname{curl} \Phi_{e}\right),
\end{aligned}
$$

where the constant $C>0$ only depends on the local geometry of $\mathcal{T}_{0}$.

The preceding inequality implies (7.8) with $C_{L ; H}=\hat{C}_{L ; H}:=\max \left(1, \frac{1}{2}(1+C)\right)$.

7.3. Remarks on the error estimator based on superconvergence. The estimator $\hat{\eta}_{R}$ guarantees sharp upper and lower bounds for the combination of the flux error and the error in the primal variable. Since $\eta_{S}$ is an error estimator designed only for the $L^{2}$-error in the primal variable, we cannot expect $\eta_{S}$ to be equivalent to $\hat{\eta}_{R}$. 
Theorem 7.3. Let $\hat{\eta}_{R}$ and $\eta_{S}$ be given by (2.2) and (2.17), respectively. Then, there exist constants $0<c_{S ; R} \leq C_{S ; R}$, independent of the refinement level, such that

$$
c_{S ; R} \eta_{S}^{2} \leq \sum_{T \in \mathcal{T}_{k}} h_{T}^{2}\left\|\mathbf{j}_{h}\right\|_{0 ; T}^{2} \leq C_{S ; R} \eta_{S}^{2}
$$

Proof. We first establish an upper bound for $h_{e}\left\|\left[u_{h}\right]_{J}^{2}\right\|_{0 ; e}$ if $e=\partial T_{1} \cap \partial T_{2}$ is an interior edge. For that purpose we denote by $\mathbf{q}_{e} \in R T_{0}\left(\Omega ; \mathcal{T}_{k}\right)$ the nodal basis field associated with $e$, i.e., $\left.\mathbf{n}_{e} \cdot \mathbf{q}_{e}\right|_{e^{\prime}}=\delta_{e, e^{\prime}}$. It follows that

$$
\begin{aligned}
h_{e}\left\|\left[u_{h}\right]_{J}\right\|_{0 ; e}^{2} & =h_{e} \int_{e}\left[u_{h}\right]_{J}\left[u_{h}\right]_{J} \mathbf{q}_{e} \cdot \mathbf{n}_{e} d \sigma=h_{e} \int_{\Omega}\left[u_{h}\right]_{J} \mathbf{j}_{h} \cdot \mathbf{q}_{e} d x \\
& \leq C\left(h_{T_{1}}^{2}\left\|\mathbf{j}_{h}\right\|_{0 ; T_{1}}^{2}+h_{T_{2}}^{2}\left\|\mathbf{j}_{h}\right\|_{0 ; T_{2}}^{2}\right),
\end{aligned}
$$

where $C>0$ is independent of $e \in \mathcal{E}_{k}$. Consequently

$$
\eta_{S ; T}^{2} \leq C \sum_{T^{\prime} \in D_{T}} h_{T^{\prime}}^{2}\left\|\mathbf{j}_{h}\right\|_{0 ; T^{\prime}}^{2}, \quad T \in \mathcal{T}_{k}
$$

where $D_{T}:=\left\{T^{\prime} \in \mathcal{T}_{k} \mid \partial T^{\prime} \cap \partial T \in \mathcal{E}_{k}\right\}$ is the union of all triangles sharing an edge with $T$. Summing over all $T \in \mathcal{T}_{k}$ and observing (1.6) gives the first inequality in (7.9).

On the other hand, to prove the second part of (7.9) we assume $\mathbf{q} \in R T_{0}(T)$ with $\mathbf{q}=\left.\mathbf{j}_{h}\right|_{T}, T \in \mathcal{T}_{k}$. Then, we get

$$
\begin{aligned}
\left\|\mathbf{j}_{h}\right\|_{0 ; T}^{2} & =\int_{T} \operatorname{div} \mathbf{q} u_{h} d x-\int_{\partial T} \lambda_{h} \mathbf{q} \cdot \mathbf{n} d \sigma=\int_{\partial T}\left(\left.u_{h}\right|_{T}-\hat{u}_{h}\right) \mathbf{q} \cdot \mathbf{n} d \sigma \\
& \leq C h_{T}^{-1}\left\|u_{h}-\hat{u}_{h}\right\|_{0 ; T}\left\|\mathbf{j}_{h}\right\|_{0 ; T},
\end{aligned}
$$

where $C>0$ is independent of $T \in \mathcal{T}_{k}$. Summing over $T \in \mathcal{T}_{k}$ and following the reasoning in the proof of Theorem 2.5 gives the assertion.

We will now show that $\left\|\mathbf{j}_{h}-K \mathbf{j}_{h}\right\|_{0 ; T}, T \in \mathcal{T}_{k}$, is equivalent to some other part of $\hat{\eta}_{R ; T}$. This, combined with the previous result, gives global equivalence of the error estimators $\hat{\eta}_{S}$ and $\hat{\eta}_{R}$.

Theorem 7.4. Let the operator $K$ be given by (2.19a) and (2.19b). Then, there exist constants $0<c_{K ; R} \leq C_{K ; R}$, depending only on the local geometry of $\mathcal{T}_{0}$, such that

$$
c_{K ; R}\left\|\mathbf{j}_{h}-K \mathbf{j}_{h}\right\|_{0 ; T}^{2} \leq \sum_{i=1}^{3} w_{i}\left\|\operatorname{curl} \varphi_{e_{i}}\right\|_{\text {div }}^{2} \leq C_{K ; R}\left\|\mathbf{j}_{h}-K \mathbf{j}_{h}\right\|_{0 ; T}^{2}, \quad T \in \mathcal{T}_{k}
$$

Proof. We consider a triangle $T \in \mathcal{T}_{k}$ with interior edges. Then (1.5), (7.6), and straightforward computations yield

$$
\begin{aligned}
& \| \mathbf{j}_{h}- K \mathbf{j}_{h} \|_{0 ; T}^{2} \\
&=\frac{1}{3}|T| \sum_{i=1}^{3}\left(\left(\left.\mathbf{t} \cdot\left(\mathbf{j}_{h}-K \mathbf{j}_{h}\right)\right|_{T}\left(m_{e_{i}}\right)\right)^{2}+\left(\left.\mathbf{n} \cdot\left(\mathbf{j}_{h}-K \mathbf{j}_{h}\right)\right|_{T}\left(m_{e_{i}}\right)\right)^{2}\right) \\
&=\frac{1}{12}|T| \sum_{i=1}^{3}\left(\left[\mathbf{j}_{h}\right]_{J}\left(m_{e_{i}}\right) \cdot \mathbf{t}\right)^{2} \leq \frac{3}{8} \kappa_{1} c_{4}^{2} \sum_{i=1}^{3} w_{i}\left\|\operatorname{curl} \varphi_{e_{i}}\right\|_{\text {div }}^{2}, \\
&\left\|\mathbf{j}_{h}-K \mathbf{j}_{h}\right\|_{0 ; T}^{2} \geq \frac{3}{8} \kappa_{0} \hat{c}_{4}^{2} \sum_{i=1}^{3} w_{i}\left\|\operatorname{curl} \varphi_{e_{i}}\right\|_{\text {div }}^{2} \cdot
\end{aligned}
$$


Corollary 7.5. Let $\hat{\eta}_{R}$ and $\hat{\eta}_{S}$ be given by (2.2) and (2.20), respectively. Then, there exist constants $0<\hat{c}_{S ; R} \leq \hat{C}_{S ; R}$, independent of the refinement level, such that

$$
\hat{c}_{S ; R} \hat{\eta}_{S}^{2} \leq \hat{\eta}_{R}^{2} \leq \hat{C}_{S ; R} \hat{\eta}_{S}^{2}
$$

Proof. The proof is an immediate consequence of Theorem 7.3 and Theorem 7.4.

We note that (7.11) only provides global equivalence of $\hat{\eta}_{S}$ and $\hat{\eta}_{R}$. However, local equivalence can be obtained, if we use $\left\|u_{h}-\hat{u}_{h}\right\|_{0}^{2}$ instead of $\sum_{e \in \mathcal{E}_{k}} h_{e}\left\|\left[u_{h}\right]_{J}\right\|_{0 ; e}^{2}$.

\section{ACKNOWLEGDMENT}

The authors are grateful to the referee for very valuable suggestions helping to improve the paper.

\section{REFERENCES}

[1] B. Achchab, A.Agouzal, J. Baranger, and J. Maitre, Estimateur d'erreur a posteriori hiérarchique. Application aux éléments finis mixtes, Numer. Math. 80 (1998), 159-179. CMP 49:01

[2] D. Arnold And F. BrezzI, Mixed and nonconforming finite element methods: Implementation, post-processing and error estimates, RAIRO Modél. Math. Anal. Numér. 19 (1985), pp. 7-35. MR 87g:65176

[3] I. BABušKa And W. Rheinboldt, Error estimates for adaptive finite element computations, SIAM J. Numer. Anal., 15 (1978), pp. 736-754. MR 58:3400

[4] — A posteriori error estimates for the finite element method, Int. J. Numer. Methods Eng., 12 (1978), pp. 1597-1615.

[5] R. BANK, A. Sherman, AND A. WeISER, Refinement algorithm and data structures for regular local mesh refinement, in Scientific Computing, R. Stepleman et al., ed., vol. 44, IMACS North-Holland, Amsterdam, 1983, pp. 3-17. MR 85i:00014

[6] R. BAnK And A. Weiser, Some a posteriori error estimators for elliptic partial differential equations, Math. Comp., 44 (1985), pp. 283-301. MR 86g:65207

[7] R. Becker and R. RAnNacher, Weighted a posteriori error control in FE methods, Tech. Report 96 - 1, SFB 359 - Universität Heidelberg, January 1996.

[8] F. Bornemann, B. Erdmann, and R. Kornhuber, A posteriori error estimates for elliptic problems in two and three spaces dimensions, SIAM J. Numer. Anal., 33 (1996), pp. 11881204 MR 98a:65161

[9] D. Braess, O. KlaAs, R. Niekamp, E. Stein, and F. Wobschal, Error indicators for mixed finite elements in 2-dimensional linear elasticity, Comput. Methods Appl. Mech. Eng., 127, (1995), pp. 345-356 MR 96h:73045

[10] D. Braess and R. Verfürth, A posteriori error estimators for the Raviart-Thomas element, SIAM J. Numer. Anal., 33 (1996), pp. 2431-2444 MR 97m:65201

[11] J. BRANDTS, Superconvergence and a posteriori error estimation for triangular mixed finite elements, Numer. Math., 68 (1994), pp. 311-324. MR 97a:65162

[12] F. Brezzi And M. Fortin, Mixed and hybrid finite element methods, Springer-Verlag, New York, 1991. MR 92d:65187

[13] P. ClÉment, Approximation by finite element functions using local regularization, RAIRO Anal. Numér., 2 (1975), pp. 77-84. MR 53:4569

[14] P. Deuflhard, P. Leinen, and H. Yserentant, Concepts of an adaptive hierarchical finite element code, IMPACT Comput. Sci. Engrg., 1 (1989), pp. 3-35.

[15] R. Duran And R. Rodriguez, On the asymptotic exactness of Bank-Weiser's estimator, Numer. Math., 62 (1992), pp. 297-303. MR 93e:65135

[16] K. ERIKSSOn And C. Johnson, An adaptive finite element method for linear elliptic problems, Math. Comput., 50 (1988), pp. 361-383.

[17] R. EWING AND J. WANG, Analysis of multilevel decomposition iterative methods for mixed finite element methods, RAIRO Modél. Math. Anal. Numér. 28 (1994), pp. 377-398. MR 95e:65099 
[18] R. Hoppe And B. Wohlmuth, Adaptive multilevel techniques for mixed finite element discretizations of elliptic boundary value problems, SIAM J. Numer. Anal., 34 (1997), pp. 16581681. MR 98e:65095

[19] — Efficient numerical solution of mixed finite element discretizations by adaptive multilevel methods, Appl. Math., 40 (1995), pp. 227-248. MR 96b:65111

[20] - Multilevel iterative solution and adaptive mesh refinement for mixed finite element discretizations, Appl. Num. Math., 23 (1997), pp. 97-117 MR 98g:65109

[21] — Element-oriented and edge-oriented local error estimators for nonconforming finite element methods, RAIRO Modél. Math. Anal. Numér. 30 (1996), pp. 237-263. MR 97e:65124

[22] C. Johnson, Numerical Solution of Partial Differential Equations by the Finite Element Method, no. 101, Cambridge University Press, Cambridge, 1987. MR 89b:65003a

[23] C. Johnson and P. Hansbo, Adaptive finite element methods in computational mechanics, Comp. Meth. Appl. Mech. Eng., 101 (1992), pp. 143-181. MR 93m:65157

[24] R. Rodriguez, Some remarks on the Zienkiewicz-Zhu estimator, Numer. Meth. for PDE, 10 (1994), pp. 625-636. MR 95e:65103

[25] T. Strouboulis And J. OdEn, A posteriori error estimation of finite element approximations in fluid dynamics, Comp. Meth. Appl. Mech. Engrg., 78 (1990), pp. 201-242. MR 91a:76066

[26] B. Szabó and I. BabušKa, Finite Element Analysis, Wiley, New York, 1991. MR 93f:73001

[27] R. Verfürth, A Review of A Posteriori Error Estimation and Adaptive Mesh-Refinement Techniques, Wiley-Teubner, Chichester, 1996.

[28] — A posteriori error estimators for the Stokes equations, Numer. Math., 55 (1989), pp. 309-325. MR 90d:65187

[29] — A posteriori error estimates for nonlinear problems. Finite element discretizations of elliptic equations, Math. Comput., 62 (1994), pp. 445-475. MR 94j:65136

[30] — A posteriori error estimation and adaptive mesh-refinement techniques, J. Comp. Appl. Math., 50 (1994), pp. 67-83. MR 95c:65171

[31] B. Wohlmuth, Adaptive Multilevel-Finite-Elemente Methoden zur Lösung elliptischer Randwertprobleme, $\mathrm{PhD}$ thesis, TU München, 1995.

[32] J. Zhu and O. Zienkiewicz, Adaptive techniques in the finite element method, Commun. Appl. Numer. Methods, 4 (1988), pp. 197-204.

[33] O. Zienkiewicz and R. TAYlor, The Finite Element Method, vol. 1, Mc Graw-Hill, London, 1989.

[34] O. Zienkiewicz AND J. ZHU, A simple error estimator and adaptive procedure for practical engineering analysis, J. Numer. Meth. Eng., 28 (1987), pp. 28-39.

Math. Institute, University of Augsburg, D-86135 Augsburg, Germany

E-mail address: wohlmuth@math.uni-augsburg.de

Math. Institute, University of Augsburg, D-86135 Augsburg, Germany

E-mail address: hoppe@math.uni-augsburg.de 